\title{
EMPIRICAL MEASURES: REGULARITY IS A COUNTER-CURSE TO DIMENSIONALITY
}

\author{
Benô̂t R. KLOECKNER*
}

\begin{abstract}
We propose a "decomposition method" to prove non-asymptotic bound for the convergence of empirical measures in various dual norms. The main point is to show that if one measures convergence in duality with sufficiently regular observables, the convergence is much faster than for, say, merely Lipschitz observables. Actually, assuming $s$ derivatives with $s>d / 2$ ( $d$ the dimension) ensures an optimal rate of convergence of $1 / \sqrt{n}$ ( $n$ the number of samples). The method is flexible enough to apply to Markov chains which satisfy a geometric contraction hypothesis, assuming neither stationarity nor reversibility, with the same convergence speed up to a power of logarithm factor. Our results are stated as controls of the expected distance between the empirical measure and its limit, but we explain briefly how the classical method of bounded difference can be used to deduce concentration estimates.
\end{abstract}

Mathematics Subject Classification. 60B10, 60J05, 62E17, 49Q20.

Received June 26, 2018. Accepted November 6, 2019.

\section{INTRODUCTION}

\subsection{Empirical measures and quadrature}

Consider a discrete-time stochastic process $\left(X_{k}\right)_{k \geq 0}$ taking its values in some domain $\Omega \subset \mathbb{R}^{d}$. We are concerned with the random atomic measure

$$
\hat{\mu}_{n}=\frac{1}{n} \sum_{k=1}^{n} \delta_{X_{k}},
$$

called the empirical measure of the process, and its convergence. We shall either assume that the $\left(X_{k}\right)_{k \geq 0}$ are independent identically distributed of some law $\mu$, or assume they form a Markov chain with weak long-range dependence and convergence of the law of $X_{k}$ to $\mu$ as $k \rightarrow \infty$.

To quantify the convergence, we are interested in distances on the set $\mathcal{P}(\Omega)$ of probability measures defined by duality. Given a class $\mathscr{F}$ of functions $f: \Omega \rightarrow \mathbb{R}$ (sometimes called "test functions" or "observables"), one

Keywords and phrases: Concentration, dual norms, empirical measure, Markov chains, non-asymptotic bounds, Wasserstein metric.

Université Paris-Est, Laboratoire d'Analyse et de Matématiques Appliquées (UMR 8050), UPEM, UPEC, CNRS, 94010 Créteil, France.

* Corresponding author: benoit.kloeckner@u-pec.fr 
defines for $\nu_{0}, \nu_{1} \in \mathcal{P}(\Omega)$ :

$$
\left\|\nu_{0}-\nu_{1}\right\|_{\mathscr{F}}=\sup _{f \in \mathscr{F}}\left|\nu_{0}(f)-\nu_{1}(f)\right|
$$

(note that we write indifferently $\nu_{0}(f)$ or $\left.\int f \mathrm{~d} \nu_{0}\right)$.

One particularly important case is obtained by taking $\mathscr{F}=\operatorname{Lip}_{1}(\Omega)$, the set of 1-Lipschitz functions. The corresponding metric is the 1 -Wasserstein metric $\mathrm{W}_{1}=\|\cdot\|_{\mathrm{Lip}_{1}}$, which by virtue of Kantorovich duality (see e.g. [10], Thm. 11.8.2) can be written equivalently as

$$
\mathrm{W}_{1}\left(\nu_{0}, \nu_{1}\right):=\inf _{X \sim \nu_{0}, Y \sim \nu_{1}} \mathbb{E}[\|X-Y\|]
$$

here $\|\cdot\|$ here is the Euclidean norm and the infimum is over all pairs of random variable with the given measures as individual laws. It is long-known [1] that, when the $\left(X_{k}\right)_{k \geq 0}$ are independent and uniformly distributed on $[0,1]^{d}$, we have

$$
\mathbb{E}\left[\mathrm{W}_{1}\left(\hat{\mu}_{n}, \lambda\right)\right] \asymp \begin{cases}\frac{1}{\sqrt{n}} & \text { if } d=1, \\ \sqrt{\frac{\log n}{n}} & \text { if } d=2, \\ \frac{1}{n^{\frac{1}{d}}} & \text { if } d \geq 3\end{cases}
$$

where $\asymp$ expresses upper and lower bounds up to multiplicative constants and $\lambda$ denotes the Lebesgue measure. This problem and generalizations have been studied in several works, e.g. $[2,3,9,12,26,27,30]$.

The bounds (1.2) are interesting theoretically, but are rather disappointing for the practical application to quadrature. Computations of integrals are in many cases impractical using deterministic methods, and one often has to resort to Monte Carlo methods, i.e. approximate the unknown $\mu(f)$ by $\hat{\mu}_{n}(f)$. When one has to compute the integrals of a large number of functions $\left(f_{m}\right)_{1 \leq m \leq M}$ with respect to a fixed measure $\mu$, one would rather draw the random quadrature points $X_{1}, \ldots, X_{k}$ once and for all, and use them for all functions $f_{m}$; while usual Monte Carlo bound will ensure each individual estimate $\hat{\mu}_{n}\left(f_{m}\right)$ has small probability to be far from $\mu\left(f_{m}\right)$, if $M$ is large compared to $n$ these bounds will not ensure that all estimates are good with high probability. On the contrary, convergence in $\mathrm{W}_{1}$ (or in duality with some other class $\mathscr{F}$ ) ensures good estimates simultaneously for all $f_{m}$, as long as they belong to the given class, independently of $M$. This makes such convergence potentially useful; but the rate given above, $n^{-\frac{1}{d}}$, is hopelessly slow in high dimension which is precisely the setting where Monte Carlo methods are most needed. We shall prove that if the functions of interest are regular enough, then this "curse of dimensionality" can be overcome. We shall be interested in the duality with $\mathcal{C}_{1}^{\text {s }}$ the set of functions with $\mathcal{C}^{\mathrm{s}}$ norm at most 1 (precise definitions are given below; when $s=1$ this is the set of 1-Lipschitz functions); but other spaces could be considered, e.g. Sobolev or Besov spaces. An approach similar in spirit, but in fact quite different, is developped by Berthet and Weed in [31]: they show that good estimators (constructed from a sample of i.i.d. random variables with law $\mu$, but different from the empirical measure) exist for probability measures with smooth enough density. This is quite orthogonal to our setting, where the measure is arbitrary and the observables are smooth.

Another issue is that in many cases, drawing independent samples $\left(X_{k}\right)_{k \geq 0}$ of law $\mu$ is not feasible, and one is lead to instead rely on a Markov chain having $\mu$ as its stationary measure; this is the Markov Chain Monte Carlo method (MCMC). While the empirical measure of Markov chains have been considered by Fournier and Guillin [12], these authors need quite strong assumptions: a spectral gap in the $L^{2}$ space (or similarly large spaces), and a "warm start" hypothesis ( $X_{0}$ should have a law absolutely continuous with respect to $\mu$ ). In good cases, one 
can achieve this by a burn-in period (start with arbitrary $X_{0}$, and consider $\left(X_{k_{0}+k}\right)_{k \geq 0}$ for some large $\left.k_{0}\right)$; but in some cases, each $X_{k}$ has a singular law with respect to $\mu$ (for example the natural random walk generated by an Iterated Function System). We shall consider Markov chains satisfying a certain geometric contraction property, but again the method can certainly be adapted to other assumptions.

\subsection{Markov chains}

Our main result handles Markov chains of arbitrary starting distribution and with a spectral gap in Lip (e.g. positively curved chains in the sense of Ollivier [23]).

Theorem 1.1. Assume that $\left(X_{k}\right)_{k \geq 0}$ is a Markov chain defined on a bounded domain $\Omega$ of $\mathbb{R}^{d}$, whose iterated transition kernel $\left(m_{x}^{t}\right)_{x \in \Omega, t \in \mathbb{N}}$ defined by

$$
m_{x}^{t}(A)=\mathbb{P}\left(X_{k+t} \in A \mid X_{k}=x\right)
$$

is exponentially contracting in the Wasserstein metric $\mathrm{W}_{1}$, i.e. there are constants $D \geq 1$ and $\theta \in(0,1)$ such that

$$
\mathrm{W}_{1}\left(m_{x}^{t}, m_{y}^{t}\right) \leq D \theta^{t}\|x-y\|
$$

Denote by $\mu$ the (unique) stationary measure of the transition kernel and let $s$ be a positive integer.

Then there exists a constant $C=C(\Omega, d, D, s)>0$ such that for all $n$, letting $\bar{n}=(1-\theta) n$, we have

$$
\mathbb{E}\left[\left\|\hat{\mu}_{n}-\mu\right\|_{\mathcal{C}_{1}^{\mathrm{s}}}\right] \leq C \begin{cases}\frac{(\log \bar{n})^{\frac{d}{2 s+1}}}{\sqrt{\bar{n}}} & \text { when } 2 s>d \\ \frac{\log \bar{n}}{\sqrt{\bar{n}}} & \text { when } 2 s=d \\ \frac{(\log \bar{n})^{d-2 s+\frac{s}{d}}}{\bar{n}^{\frac{s}{d}}} & \text { when } 2 s<d .\end{cases}
$$

Note that the hypothesis that the Markov chain is exponentially contracting corresponds to its Markov operator (acting on the space of Lipschitz functions) having a spectral gap. Let us stress two strengths of this result:

- for $s=1$, recalling $\|\cdot\|_{\mathcal{C}_{1}^{1}}=\|\cdot\|_{\mathrm{Lip}_{1}}=\mathrm{W}_{1}$, the bounds are only a power of logarithm factor away from the optimal bounds for i.i.d. random variables,

- for $s$ large enough, we almost obtain the optimal convergence rate $\asymp 1 / \sqrt{n}$

- we assume neither reversibility, stationarity, nor warm start hypotheses (the distribution of $X_{0}$ can be arbitrary),

- the rate of convergence does not depend on the specific feature of the Markov chain, only on $D$ and $\theta$.

Note that for fixed $\theta, \bar{n}$ has the same order than $n$, but if $\theta$ is close to $1,1 /(1-\theta)$ is the typical time scale for the decay of correlations. One thus cannot expect less than $(1-\theta) n$ Markov samples to achieve the bound obtained for $n$ independent samples.

Examples of Markov chains which are exponentially contracting in $\mathrm{W}_{1}$ (equivalently, that have a spectral gap in the space of Lipschitz observables) are numerous (and include many Markov chains that fail to converge to the stationary measure in the total variation distance); it is a slightly more general condition than "positive curvature" in the sense of Ollivier [23] (close to the "one-step contracting" condition in [7]), see e.g. [15] and [17] for concrete examples, or in the context of dynamical systems [18] and [16]. The case of continuous-time processes also produce many relevant examples, see e.g. [11] and [22]. 
Under the assumption of Theorem 1.1, it is well-known that uniform concentration and bias estimates hold:

$$
\sup _{f \in \mathscr{F}} \mathbb{P}\left(\left|\hat{\mu}_{n}(f)-\mu(f)\right|>\varepsilon\right) \rightarrow 0 \quad \text { and } \quad \sup _{f \in \mathscr{F}} \mathbb{E}\left[\left|\hat{\mu}_{n}(f)-\mu(f)\right|\right] \rightarrow 0
$$

with $\mathscr{F}=\operatorname{Lip}_{1}$ (or any smaller class); see [15] and [24] for explicit rates, in particular Gaussian concentration rates (i.e. of the form $2 e^{-c n \varepsilon^{2}}$ ) when $\varepsilon$ is small enough.

The problem of convergence in duality to the class $\mathscr{F}$ is thus to invert the supremum and the probability (or expectation), to bound from above

$$
\mathbb{P}\left(\sup _{f \in \mathscr{F}}\left|\hat{\mu}_{n}(f)-\mu(f)\right|>\varepsilon\right) \quad \text { or } \quad \mathbb{E}\left[\sup _{f \in \mathscr{F}}\left|\hat{\mu}_{n}(f)-\mu(f)\right|\right]
$$

We shall disregard the potential issue of non-measurability: as we shall only deal with classes $\mathscr{F}$ having a countable subset which is dense in the uniform norm, we can always replace the supremum with a supremum over a countable set of functions.

The idea of the proof of Theorem 1.1 is to take an arbitrary $f \in \mathcal{C}_{1}^{\mathrm{s}}(\Omega)$ and decompose it using Fourier series. The regularity hypothesis gives us a control on both the uniform approximation by a truncated Fourier series, and on the Fourier coefficients. Combining these controls, we bound from above $\left|\hat{\mu}_{n}(f)-\mu(f)\right|$ by a quantity that does not depend on $f$ at all, but depends on the Fourier basis elements $\left(e_{k}\right)_{k \in \mathbb{Z}^{d}}$ up to some index size. Taking a supremum and an expectation, this leaves us with the simple task to optimize where to truncate the Fourier series.

This decomposition method can in principle be used under various assumptions on the process $\left(X_{k}\right)_{k \geq 0}$, the point being to identify a decomposition suited to the assumption; in particular, one can easily adapt the method to study geometrically ergodic Markov chains. I chose to present Theorem $1.1 \mathrm{in}$ part because its hypothesis is relevant to several Markov chains I am interested in, and in part because it presents specific difficulties: a blunt computation leads to non-optimal powers of $n$. To obtain good rates, we translate the contraction hypothesis to frame part of the argument in the space $\mathrm{Hol}_{\alpha}$, where the Fourier basis has smaller norm; and instead of bounding the Fourier coefficients of a Lipschitz function directly, we use Parceval's formula and the injection $\mathcal{C}^{\mathrm{s}} \rightarrow H^{s}$ which turns out to give a better estimate. Another functional decomposition, and another path in computations might improve the power in the logarithmic factor.

We restrict to the compact case, but the method can in principle be adapted, or truncation argument be used, to deal with non-compactly supported measure.

In order to introduce the decomposition method and show its flexibility, we shall state some simpler results below.

\subsection{Explicit bounds in the i.i.d case, for the Wasserstein metric}

The decomposition method enables one to get a very explicit version of (1.2) with a few computations but very little sophistication.

Theorem 1.2. If $\mu$ is any probability measure on $[0,1]^{d}$ and $\left(X_{k}\right)_{k \geq 0}$ are i.i.d. random variable with law $\mu$, then for all $n \in \mathbb{N}$ we have

$$
\mathbb{E}\left[\mathrm{W}_{1}\left(\hat{\mu}_{n}, \mu\right)\right] \leq \begin{cases}\frac{1}{2(\sqrt{2}-1)} \cdot \frac{1}{\sqrt{n}} & \text { when } d=1, \\ \frac{\log _{2}(n)+8}{\sqrt{8 n}} & \text { when } d=2, \\ \frac{C_{d}}{n^{\frac{1}{d}}} & \text { when } d \geq 3\end{cases}
$$


where $C_{3} \leq 6.3, C_{d} \leq 3 \sqrt{d}$ for all $d \geq 4$, and $C_{d} / \sqrt{d} \rightarrow 2$ as $d \rightarrow \infty$.

The order of magnitude of these bounds is sharp in many regimes:

- in dimension 1 , the order of magnitude $1 / \sqrt{n}$ is optimal; however the constant $1 /(2(\sqrt{2}-1)$ ) is not asymptotically optimal when $\mu$ is Lebesgue measure,

- when $d=2$ and $\mu$ is Lebesgue measure, as previously mentioned the correct order is $\sqrt{\log n / n}$, however see Theorem 1.3 below: the same method provides similar upper bounds for some fractal measures, and when the fractal dimension is 2 the rate $\log (n) / \sqrt{n}$ is actually sharp.

- when $d \geq 3$, both orders of magnitude $n^{-1 / d}$ as $n \rightarrow \infty$ and $\sqrt{d}$ as $d \rightarrow \infty$ are sharp up to multiplicative constants (see Rem. 2.2). The asymptotic constant 2 is certainly quite larger than the asymptotic constant

$$
\lim _{d \rightarrow \infty} \lim _{n \rightarrow \infty} \frac{n^{\frac{1}{d}}}{\sqrt{d}} \mathbb{E}\left[\mathrm{W}_{1}\left(\hat{\mu}_{n}, \lambda\right)\right]
$$

which has been computed for the related, but slightly different matching problem by Talagrand [26]; but our bound holds for all $n$ and all $d$ (and also all $\mu$ ). An even more general bound has been given by Boissard and Le Gouic [3], but their constant is larger by a factor approximately 10.

Let us stress that the main purpose of this result will be to expose our method in an elementary setting: indeed many previous similar bounds are available in this case. For example more general non-asymptotic results have been obtained by Fournier and Guillin [12], building on previous work by Dereich et al. [9]. They are more general in that they consider $q$-Wasserstein metric for any $q>0$ (while we will only be able to consider $q \leq 1$ ), and apply to non-compactly supported measures $\mu$ under moment assumptions. However their constants, though non-asymptotic, have not been made explicit, and their behavior when the dimension grows has not been studied.

The available methods are able to prove similar upper bounds for some fractal measures; we shall prove that in some cases, these upper bound are sharp even at the critical dimension.

Theorem 1.3. Let $\mu$ be the uniform measure on a self-similar, strongly separated fractal (see definitions in Sect. 2.4) and let $\left(X_{k}\right)_{k \geq 0}$ be i.i.d. random variables of law $\mu$ and $q \in(0,1]$. Let $D$ be the fractal dimension of $\mu$. Then

$$
\mathbb{E}\left[\mathrm{W}_{q}\left(\hat{\mu}_{n}, \mu\right)\right] \asymp \begin{cases}\frac{1}{\sqrt{n}} & \text { if } D<2 q, \\ \frac{\log n}{\sqrt{n}} & \text { if } D=2 q, \\ \frac{1}{n^{\frac{q}{D}}} & \text { if } D>2 q .\end{cases}
$$

The sharpness of the bound $\log n / \sqrt{n}$ in the critical case here contrasts with the case of the uniform measure on the square (1.2). Methods that rely on a multiscale decomposition in any way applicable to self-similar fractal measure thus cannot yield the optimal estimate of [1] in the case of Lebesgue measure.

Note that a self-similar fractal of dimension 2 with the strong separation condition does not admit a biLipschitz embedding in $\mathbb{R}^{2}$, and the following question, already stressed in [12], is left open: is it true that $\mathbb{E}\left[\mathrm{W}_{1}\left(\hat{\mu}_{n}, \mu\right)\right] \lesssim \sqrt{\log (n) / n}$ for all $\mu \in \mathcal{P}\left([0,1]^{2}\right) ?$

\subsection{Regular observables and independent samples}

In the i.i.d. case, we can improve Theorem 1.1 by removing most of the logarithmic factors. 
Theorem 1.4. If $\mu$ is any probability measure on $[0,1]^{d}$ and $\left(X_{k}\right)_{k \geq 0}$ are i.i.d. random variable with law $\mu$, then for all $s \geq 1$, for some constant $C=C(d, s)>0$ (not depending upon $\mu$ ), and all integer $n \geq 2$ we have

$$
\mathbb{E}\left[\left\|\hat{\mu}_{n}-\mu\right\|_{\mathcal{C}_{1}^{\mathrm{s}}}\right] \leq C \begin{cases}\frac{1}{\sqrt{n}} & \text { when } s>\frac{d}{2}, \\ \frac{\log n}{\sqrt{n}} & \text { when } s=\frac{d}{2}, \\ \frac{1}{n^{s / d}} & \text { when } s<\frac{d}{2} .\end{cases}
$$

It is possible to prove this result with previous, more classical methods. Indeed, combining the "entropy bound" for the class $\mathcal{C}_{1}^{\mathrm{s}}$ ([28, Thm. 2.7.1]) and the "chaining method" (see e.g. [29, Ex 5.11, p. 138]) leads to Theorem 1.4; I am indebted to Jonathan Weed for pointing this out to me. The proof by the decomposition method we provide here is very simple, but non-elementary as it relies on a wavelet decomposition. It is wellknown that all functions in $\mathcal{C}_{1}^{\mathrm{s}}$ can be written as a linear combination of a few elements of a wavelet basis, with small coefficients, up to a small error. Then controlling $\left|\hat{\mu}_{n}(f)-\mu(f)\right|$ for all $f \in \mathcal{C}_{1}^{\text {s }}$ simultaneously reduces to controlling this quantity for the few needed elements of the wavelet basis.

\subsection{Concentration inequalities}

Up to know, we have restricted to estimates on the expectation, while in many practical situations one would need concentration estimates. This is in fact not a restriction, as we shall explain briefly in Section 5: the classical bounded difference method enable one to get concentration near the expectation. In particular, we get the following.

Corollary 1.5. Under the assumptions of Theorem 1.1, there exist $\epsilon=\epsilon(\theta, D, \operatorname{diam} \Omega)>0, C=C(\Omega, d, D, \theta)>$ 0 such that for all $n \geq N$ and all $M \geq C$ we have:

- when $s>d / 2$

$$
\mathbb{P}\left[\left\|\hat{\mu}_{n}-\mu\right\|_{\mathcal{C}_{1}^{\S}} \geq M \frac{(\log n)^{\frac{d}{2 s+1}}}{\sqrt{n}}\right] \leq e^{-\epsilon(M-C)^{2}(\log n)^{\frac{2 d}{2 s+1}}},
$$

- when $s=d / 2$

$$
\mathbb{P}\left[\left\|\hat{\mu}_{n}-\mu\right\|_{\mathcal{C}_{1}^{s}} \geq M \frac{\log n}{\sqrt{n}}\right] \leq e^{-\epsilon(M-C)^{2}(\log n)^{2}},
$$

- when $s<d / 2$

$$
\mathbb{P}\left[\left\|\hat{\mu}_{n}-\mu\right\|_{\mathcal{C}_{1}^{s}} \geq M \frac{(\log n)^{d-2 s+\frac{s}{d}}}{n^{\frac{s}{d}}}\right] \leq e^{-\epsilon(M-C)^{2} n^{1-2 s / d}} .
$$

(The last inequality is not optimal as we relaxed the poly-logarithmic factor for simplicity.) For example, when $\frac{d}{2}<s<d-\frac{1}{2}$ we deduce that

$$
\frac{\sqrt{n}}{(\log n)^{\frac{d}{2 s+1}}}\left\|\hat{\mu}_{n}-\mu\right\|_{\mathcal{C}_{1}^{\mathrm{s}}}
$$


is bounded almost surely.

The recent article [8] gives related deviation inequalities. Compared to Corollary 1.5, on the one hand they restrict to the i.i.d. case and Wasserstein distances, but on the other hand they allow for non-compactly supported measures $\mu$ and consider $p$-Wasserstein distances with $p \geq 1$. They only make weak moment assumptions, which leads to an unavoidable polynomial part in the bounds; in the cases where both apply, our bounds above are thus stronger for fixed $M$ and large $n$. The question remains of what order of concentration one should expect in the case of a Markov chain with non compactly supported stationary measure.

Structure of the paper Sections 2-4 are independent and contain respectively the proofs of Theorems 1.2 and 1.3, 1.4, 1.1: we start with the most elementary proofs, follow with the simplest one, and end with the most sophisticated).

Section 5, dealing with concentration estimates, is mostly independent from the previous ones, which are only used to deduce Corollary 1.5 .

We shall write $a \lesssim b$ for $a \leq C b$, the dependency of the constant $C$ being left implicit unless it feels necessary; the constants denoted by $C$ will be allowed to change from line to line.

\section{WASSERSTEIN CONVERGENCE AND DYADIC DECOMPOSITION}

The goal of this section is to prove (a refinement of) Theorem 1.2. We consider a sequence $\left(X_{k}\right)_{1 \leq k}$ of independent, identically distributed random points whose common law shall be denoted by $\mu$; we assume that $\mu$ is supported on the cube $[0,1]^{d}$ and consider the convergence of the empirical measure $\hat{\mu}_{n}:=\sum_{k=1}^{n} \frac{1}{n} \delta_{X_{k}}$ in the $q$-Wasserstein distance where $q \in(0,1]$, i.e.

$$
\mathrm{W}_{q}\left(\mu_{0}, \mu_{1}\right):=\sup _{f \in \mathrm{Hol}_{1}^{q}}\left|\mu_{0}(f)-\mu_{1}(f)\right|
$$

where $\operatorname{Hol}_{1}^{q}$ is the set of functions $f:[0,1]^{d} \rightarrow \mathbb{R}$ such that for all $x, y \in[0,1]^{d}$ :

$$
|f(x)-f(y)| \leq\|x-y\|^{q}
$$

(note that this is the usual Wasserstein distance with respect to the "snowflake" metric $\|\cdot\|^{q}$, and thus benefits from all its usual properties; in particular $\mathrm{W}_{q}$ is a metric).

While we are mostly interested in the Euclidean norm $\|\cdot\|$, our method is sharper in the case of the supremum norm ${ }^{1}\|\cdot\|_{\infty}$, with respect to which the analogue of the aforementioned objects are denoted by $\mathrm{W}_{q, \infty}$ and $\mathrm{Hol}_{1}^{q, \infty}$. We will work with $\|\cdot\|_{\infty}$, and then deduce directly the corresponding result for the Euclidean norm by using that $\|\cdot\| \leq \sqrt{d}\|\cdot\|_{\infty}$ (and thus $\mathrm{W}_{q} \leq d^{\frac{q}{2}} \mathrm{~W}_{q, \infty}$ ).

Our most precise result is the following.

Theorem 2.1. For all $q \in(0,1]$ and all $n$, it holds:

$$
\mathbb{E}\left[\mathrm{W}_{q, \infty}\left(\hat{\mu}_{n}, \mu\right)\right] \leq \begin{cases}\frac{2^{\frac{d}{2}-2 q}}{1-2^{\frac{d}{2}-q}} \cdot \frac{1}{\sqrt{n}} & \text { when } d<2 q, \\ \left(2+\frac{\log _{2}(n)}{2^{q+1} q}\right) \frac{1}{\sqrt{n}} & \text { when } d=2 q, \\ 2\left(\frac{\frac{d}{2}-q}{2 q\left(1-2^{q-\frac{d}{2}}\right)}\right)^{\frac{2 q}{d}}\left(1+\frac{q}{2^{q}\left(\frac{d}{2}-q\right)}\right) \frac{1}{n^{\frac{q}{d}}} & \text { when } d>2 q .\end{cases}
$$

\footnotetext{
${ }^{1}$ The same notation is used for the uniform norm of functions, but the type of the argument will prevent any confusion.
} 
We deduce several more compact formulas below, including Theorem 1.2. Observe that for fixed $q$ and large $d$, the complicated front constant converges to 2 .

Remark 2.2. It is not difficult to see that for $\mu$ the Lebesgue measure and an optimal, deterministic approximation $\tilde{\mu}_{n}$ with $n=k^{d}$ Dirac masses, one has

$$
\mathrm{W}_{1, \infty}\left(\tilde{\mu}_{n}, \mu\right) \geq \frac{d}{(d+q) 2^{q}} \frac{1}{n^{\frac{q}{d}}}
$$

(use that the $\|\cdot\|_{\infty}$-spheres have $(d-1)$-dimensional volume equal to $2 d \times(2 r)^{d-1}$ : in the best-case scenario, all support points of $\tilde{\mu}_{n}$ are coupled exactly with $\|\cdot\|_{\infty}$-balls, all of the same radius $r_{+}$; a simple integration similar to the one below gives the claimed bound). It follows that in high dimension, for the $\ell^{\infty}$ norm and in the worst case $q=1$ our estimate is off by a factor of approximately 4 compared to a best approximation.

With the Euclidean norm, an easy lower bound in the case of the Lebesgue measure is obtained by observing similarly that a mass at most

$$
\frac{\pi^{\frac{d}{2}}}{\Gamma\left(\frac{d}{2}+1\right)} R^{d} n
$$

is at distance $R$ or less of one of the $n$ points (be they random or not). This leads, for any measure $\tilde{\mu}_{n}$ supported on $n$ points, to

$$
\mathrm{W}_{q}\left(\tilde{\mu}_{n}, \mu\right) \geq n \int_{0}^{R_{0}} d \frac{\pi^{\frac{d}{2}}}{\Gamma\left(\frac{d}{2}+1\right)} R^{d} \mathrm{~d} R=n \frac{d \pi^{\frac{d}{2}}}{(d+1) \Gamma\left(\frac{d}{2}+1\right)} R_{0}^{d+1}
$$

where $R_{0}$ is defined by $n \frac{\pi^{\frac{d}{2}}}{\Gamma\left(\frac{d}{2}+1\right)} R_{0}^{d}=1$. Finally,

$$
W_{1}\left(\tilde{\mu}_{n}, \mu\right) \geq \underbrace{\frac{d \Gamma\left(\frac{d}{2}+1\right)^{\frac{1}{d}}}{(d+1) \sqrt{\pi}}}_{\substack{\sim \sim \infty \\ d \rightarrow \sqrt{\frac{d}{2 e \pi}}}} \cdot \frac{1}{n^{\frac{1}{d}}}
$$

and again our order of magnitude $C_{d} \asymp \sqrt{d}$ is the correct one.

The results of [26] show that, at least for the bipartite matching problem, this seemingly crude lower bounds are in fact attained asymptotically, taking renormalized limits as $n \rightarrow \infty$ and then $d \rightarrow \infty$. This indicates that our constant are not optimal, and it would be interesting to have a non-asymptotic bound with optimal asymptotic behavior.

\subsection{Decomposition of Hölder functions}

The method to prove Theorem 2.1 consists in a multiscale decomposition of the functions $f \in \operatorname{Hol}_{1}^{q, \infty}$. In its spirit, it seems quite close to arguments of [3], [9] and [12]; our interest is mostly in setting this multiscale analysis in a functional decomposition framework.

We fix a positive integer $J$ to be optimized later, representing the depth of the decomposition. For each $j \in\{0, \ldots, J\}$, set $\Lambda_{j}=\{j\} \times\left\{0, \ldots, 2^{j}-1\right\}^{d}$; then define $\Lambda=\bigcup_{j=0}^{J} \Lambda_{j}$, acting as the set of indices for the decomposition.

For each $j \in\{0, \ldots, J\}$, let $\left\{C_{\lambda}: \lambda \in \Lambda_{j}\right\}$ be the regular decomposition of $[0,1]^{d}$ into cubes of side-length $2^{-j}$; the boundary points are attributed in an arbitrary (measurable) manner, with the constraint that $\left\{C_{\lambda}: \lambda \in \Lambda_{j}\right\}$ 
is a partition of $[0,1]^{d}$ that refines the previous partition $\left\{C_{\lambda}: \lambda \in \Lambda_{j-1}\right\}$. Denote by $x_{\lambda}$ the center of the cube $C_{\lambda}$, and by $\psi_{\lambda}:=\mathbf{1}_{C_{\lambda}}$ the characteristic function of $C_{\lambda}$ (so that for each $j, \sum_{\lambda \in \Lambda_{j}} \psi_{\lambda}=\mathbf{1}_{[0,1]^{d}}$ ).

Lemma 2.3. For all function $f \in \operatorname{Hol}_{1}^{q, \infty}$ and all $J$, there exist coefficients $\alpha(\lambda) \in \mathbb{R}$ such that

$$
f=\sum_{j=1}^{J} \sum_{\lambda \in \Lambda_{j}} \alpha(\lambda) \psi_{\lambda}+c+g
$$

where $c$ is a constant and $g$ is a function $[0,1]^{d} \rightarrow \mathbb{R}$, such that

$$
\begin{aligned}
|\alpha(\lambda)| & \leq 2^{-(j+1) q} \quad \forall \lambda \in \Lambda_{j} \\
\|g\|_{\infty} & \leq 2^{-(J+1) q} .
\end{aligned}
$$

Proof. Replacing $f$ with $f-c$ where $c=f\left(x_{0,0}\right)$, we assume that $f$ vanishes at the center $x_{0,0}$ of $C_{0,0}=[0,1]^{d}$. Observe that $f \in \operatorname{Hol}_{1}^{q, \infty}$ implies that $\|f\|_{\infty} \leq 2^{-q}$ and $\left|f\left(x_{\lambda}\right)\right| \leq 2^{-2 q}$ for all $\lambda \in \Lambda_{1}$.

For $\lambda \in \Lambda_{1}$, we define $\alpha(\lambda)=f\left(x_{\lambda}\right)$ and set $f_{1}=\sum_{\lambda \in \Lambda_{1}} \alpha(\lambda) \psi_{\lambda}$; we have $|\alpha(\lambda)| \leq 2^{-2 q}$, the function $f-f_{1}$ is $\mathrm{Hol}_{1}^{q, \infty}$ on $C_{\lambda}$ and vanishes at $x_{\lambda}$. Since $C_{\lambda}$ is a $\|\cdot\|_{\infty}$ ball of center $x_{\lambda}$ and radius $1 / 4$, it follows that $\left\|f-f_{1}\right\|_{\infty} \leq$ $2^{-2 q}$ on each $C_{\lambda}$, and thus on the whole of $[0,1]^{d}$. Moreover for all $\lambda \in \Lambda_{2}$ it holds $\left|\left(f-f_{1}\right)\left(x_{\lambda}\right)\right| \leq 2^{-3 q}$.

Similarly, we define $f_{j}:[0,1]^{d} \rightarrow \mathbb{R}$ recursively by setting $\alpha(\lambda)=\left(f-f_{j-1}\right)\left(x_{\lambda}\right)$ for all $\lambda \in \Lambda_{j}$ and $f_{j}=$ $f_{j-1}+\sum_{\lambda \in \Lambda_{j}} \alpha(\lambda) \psi_{\lambda}$. Then $|\alpha(\lambda)| \leq 2^{-(j+1) q}$ for all $\lambda \in \Lambda_{j}$ and $\left\|f-f_{J}\right\|_{\infty} \leq 2^{-(J+1) q}$.

\subsection{Wasserstein distance estimation}

With the notation of Lemma 2.3 , for any $f \in \mathrm{Hol}_{1}^{q}$ we have:

$$
\begin{aligned}
\left|\hat{\mu}_{n}(f)-\mu(f)\right| & \leq 2\|g\|_{\infty}+\sum_{j=1}^{J} \sum_{\lambda \in \Lambda_{j}}|\alpha(\lambda)|\left|\hat{\mu}_{n}\left(\psi_{\lambda}\right)-\mu\left(\psi_{\lambda}\right)\right| \\
& \leq 2^{1-(J+1) q}+\sum_{j=1}^{J} 2^{-(j+1) q} \sum_{\lambda \in \Lambda_{j}}\left|\hat{\mu}_{n}\left(\psi_{\lambda}\right)-\mu\left(\psi_{\lambda}\right)\right|
\end{aligned}
$$

where the last right-hand term does not depend on $f$ in any way. We can thus take a supremum and an expectation to obtain

$$
\mathbb{E}\left[\mathrm{W}_{q, \infty}\left(\hat{\mu}_{n}, \mu\right)\right] \leq 2^{1-(J+1) q}+\sum_{j=1}^{J} 2^{-(j+1) q} \sum_{\lambda \in \Lambda_{j}} \mathbb{E}\left[\left|\hat{\mu}_{n}\left(\psi_{\lambda}\right)-\mu\left(\psi_{\lambda}\right)\right|\right]
$$

Remark 2.4. This is the core of the decomposition method. Observe that we used no hypothesis on the $\left(X_{k}\right)$ yet; any stochastic process for which one can control $\mathbb{E}\left[\left|\hat{\mu}_{n}\left(\psi_{\lambda}\right)-\mu\left(\psi_{\lambda}\right)\right|\right]$ can be applied the method.

Setting $p_{\lambda}=\mu\left(\psi_{\lambda}\right)$, the random variable $n \hat{\mu}_{n}\left(\psi_{\lambda}\right)$ is binomial of parameters $n$ and $p_{\lambda}$. A standard estimation of the mean absolute deviation yields

$$
\begin{aligned}
\mathbb{E}\left[\left|n \hat{\mu}_{n}\left(\psi_{\lambda}\right)-n \mu\left(\psi_{\lambda}\right)\right|\right] & \leq \sqrt{n p_{\lambda}\left(1-p_{\lambda}\right)} \\
\sum_{\lambda \in \Lambda_{j}} \mathbb{E}\left[\left|\hat{\mu}_{n}\left(\psi_{\lambda}\right)-\mu\left(\psi_{\lambda}\right)\right|\right] & \leq \frac{1}{\sqrt{n}} \sum_{\lambda \in \Lambda_{j}} \sqrt{p_{\lambda}} .
\end{aligned}
$$


By concavity of the square-root function, we have

$$
2^{-d j} \sum_{\lambda \in \Lambda_{j}} \sqrt{p_{\lambda}} \leq \sqrt{2^{-d j} \sum_{\lambda \in \Lambda_{j}} p_{\lambda}}=2^{-\frac{d j}{2}}
$$

and we deduce

$$
\begin{aligned}
\sum_{\lambda \in \Lambda_{j}} \mathbb{E}\left[\left|\hat{\mu}_{n}\left(\psi_{\lambda}\right)-\mu\left(\psi_{\lambda}\right)\right|\right] & \leq \frac{2^{\frac{d j}{2}}}{\sqrt{n}} \\
\mathbb{E}\left[\mathrm{W}_{q, \infty}\left(\hat{\mu}_{n}, \mu\right)\right] & \leq 2^{1-(J+1) q}+\sum_{j=1}^{J} \frac{2^{j\left(\frac{d}{2}-q\right)-q}}{\sqrt{n}}
\end{aligned}
$$

leaving us with the simple task to optimize the choice of $J$.

\subsection{Optimization of the depth parameter}

We shall distinguish three cases: $d<2 q, d=2 q$ and $d>2 q$. The first case is only possible for $d=1$, but we let it phrased that way because for some measures $\mu$ the dimension $d$ of the ambient space can be replaced by the "dimension" of the measure itself, see Section 2.4 for an example.

\subsubsection{Small dimension}

If $d<2 q$, then the sum in (2.3) is bounded independently of $J$ and we can let $J \rightarrow \infty$ to obtain:

$$
\begin{aligned}
\mathbb{E}\left[\mathrm{W}_{q, \infty}\left(\hat{\mu}_{n}, \mu\right)\right] & \leq \frac{2^{-q}}{\sqrt{n}} \sum_{j=1}^{\infty} 2^{j\left(\frac{d}{2}-q\right)} \\
& \leq \frac{2^{\frac{d}{2}-2 q}}{1-2^{\frac{d}{2}-q}} \cdot \frac{1}{\sqrt{n}} .
\end{aligned}
$$

In particular, for $d=1, q=1$ :

$$
\mathbb{E}\left[\mathrm{W}_{1}\left(\hat{\mu}_{n}, \mu\right)\right] \leq \frac{1}{2(\sqrt{2}-1)} \cdot \frac{1}{\sqrt{n}}
$$

Remark 2.5. For $\frac{d}{2}-q$ close to 0 , the constant in (2.4) goes to infinity; in this regime, for moderate $n$ letting $J \rightarrow \infty$ is sub-optimal and one should optimize $J$ in (2.3) as we shall do in the next cases.

\subsubsection{Critical dimension}

If $d=2 q$ (or in fact $d \leq 2 q$ ) we can rewrite (2.3) as

$$
\mathbb{E}\left[\mathrm{W}_{q, \infty}\left(\hat{\mu}_{n}, \mu\right)\right] \leq 2^{1-(J+1) q}+\frac{2^{-q} J}{\sqrt{n}} .
$$

To optimize $J$, we formally differentiate the right-hand side with respect to $J$, equate to zero and solve for $J$. Reminding that $J$ is an integer, and keeping only the leading term (when $n \rightarrow \infty$ ) to simplify, this leads us to 
choose

$$
J=\left\lfloor\frac{\log _{2} n}{2 q}\right\rfloor
$$

in particular implying $2^{1-(J+1) q} \leq 2 / \sqrt{n}$. We deduce the claimed bound

$$
\mathbb{E}\left[\mathrm{W}_{q, \infty}\left(\hat{\mu}_{n}, \mu\right)\right] \leq\left(2+\frac{\log _{2}(n)}{2^{q+1} q}\right) \frac{1}{\sqrt{n}} \lesssim \frac{\log n}{\sqrt{n}}
$$

immediately implying the bound of Theorem 1.2 for $d=2$ and $q=1$ (where a $\sqrt{2}$ comes from the comparison between the supremum and Euclidean norms):

$$
\mathbb{E}\left[\mathrm{W}_{1}\left(\hat{\mu}_{n}, \mu\right)\right] \leq \frac{\log _{2}(n)+8}{\sqrt{8 n}} .
$$

\subsubsection{Large dimension}

If $d>2 q$, equation (2.3) becomes

$$
\mathbb{E}\left[\mathrm{W}_{q, \infty}\left(\hat{\mu}_{n}, \mu\right)\right] \leq 2^{1-(J+1) q}+\frac{2^{J\left(\frac{d}{2}-q\right)}-1}{1-2^{q-\frac{d}{2}}} \cdot \frac{1}{2^{q} \sqrt{n}} \leq 2^{1-(J+1) q}+\frac{2^{J\left(\frac{d}{2}-q\right)}}{2^{q}\left(1-2^{q-\frac{d}{2}}\right)} \cdot \frac{1}{\sqrt{n}} .
$$

Following the same optimization process as in the critical dimension case, we choose $J$ such that

$$
\frac{1}{2} n^{\frac{1}{d}}\left(\frac{2 q\left(1-2^{q-\frac{d}{2}}\right)}{\frac{d}{2}-q}\right)^{\frac{2}{d}} \leq 2^{J} \leq n^{\frac{1}{d}}\left(\frac{2 q\left(1-2^{q-\frac{d}{2}}\right)}{\frac{d}{2}-q}\right)^{\frac{2}{d}}
$$

leading to

$$
\mathbb{E}\left[\mathrm{W}_{q, \infty}\left(\hat{\mu}_{n}, \mu\right)\right] \leq 2\left(\frac{\frac{d}{2}-q}{2 q\left(1-2^{q-\frac{d}{2}}\right)}\right)^{\frac{2 q}{d}}\left(1+\frac{q}{2^{q}\left(\frac{d}{2}-q\right)}\right) \frac{1}{n^{\frac{q}{d}}} .
$$

For $q=1$ and $d \geq 3$, it comes $\mathbb{E}\left[\mathrm{W}_{1, \infty}\left(\hat{\mu}_{n}, \mu\right)\right] \leq C_{d}^{\prime} n^{-\frac{1}{d}}$ where

$$
C_{d}^{\prime}=2\left(\frac{\frac{d}{2}-1}{2-2^{2-\frac{d}{2}}}\right)^{\frac{2}{d}}\left(1+\frac{1}{d-2}\right) \frac{1}{n^{\frac{1}{d}}} .
$$

We have notably $C_{4}^{\prime}=3$. Relaxing our bound for $d \geq 4$ to

$$
C_{d}^{\prime} \leq 2\left(\frac{d}{4}\right)^{\frac{2}{d}}\left(1+\frac{1}{d-2}\right)
$$

it is more easily seen that it is decreasing (and still takes the value 3 at $d=4$ ). We also see that we can take $C_{d}^{\prime} \rightarrow 2$ as $d \rightarrow \infty$. The last part of Theorem 1.2 follows with $C_{d}=\sqrt{d} C_{d}^{\prime}$, and a numerical computation shows $C_{3} \leq 6.3$.

\subsection{Self similar fractal measures}

We conclude this section with Theorem 1.3, showing that the critical case order $\log n / \sqrt{n}$ is sharp if one generalizes its scope. 


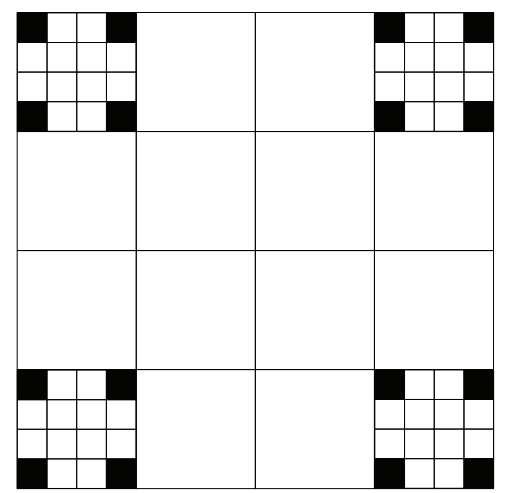

Figure 1. Second stage of the construction of the four-corners Cantor set (contained in the filled black area).

\subsubsection{Iterated function systems and fractals}

We shall consider certain fractal measures on $\mathbb{R}^{d}$. Consider an Iterated Function System (IFS) $\Phi=$ $\left(\phi_{1}, \ldots, \phi_{m}\right)$ (where $m \geq 2$ ), i.e. a collection of contractive mappings which we assume here to be similarities with equal ratios:

$$
\phi_{j}(x)=a U_{j} x+b_{j} \quad \forall x \in \mathbb{R}^{d}
$$

where $a \in(0,1)$, each $U_{j}$ is an orthogonal matrix, and the $b_{j}$ are elements of $\mathbb{R}^{d}$. It is a classical application of the Banach fixed point Theorem that there is a unique compact set $K \subset \mathbb{R}^{d}$ such that $K=\cup_{j} \phi_{j}(K)[13]$; it is called the attractor of the IFS, and is also called a self-similar fractal. There is also a unique probability measure $\mu$ such that

$$
\mu=\sum_{j=1}^{m} \frac{1}{m}\left(\phi_{j}\right)_{*} \mu
$$

(the map $\nu \mapsto \sum_{j} \frac{1}{m}\left(\phi_{j}\right)_{*} \nu$ is contracting in the complete metric $\mathrm{W}_{1}$, so that it has a unique fixed point; actually this argument provides a unique fixed point among measures of finite first moment, but it can be shown that no other fixed probability measure exists). This measure shall be called the uniform measure on $K$. We assume further that $\Phi$ satisfies the following strong separation property: there is a compact set $K^{\prime} \subset \mathbb{R}^{d}$ such that for all $j, \phi_{j}\left(K^{\prime}\right) \subset K^{\prime}$ and for all $j \neq \ell, \phi_{j}\left(K^{\prime}\right) \cap \phi_{\ell}\left(K^{\prime}\right)=\varnothing$ (this is in fact equivalent to $\phi_{j}(K) \cap \phi_{\ell}(K)=\varnothing$ for all $j \neq \ell$, but has the advantage of being easy to check without constructing $K$ ). Examples of sets $K$ obtained as attractor of such an IFS are numerous; for example the classical middle-third Cantor set, or the four-corner cantor set of the plane (Fig. 1).

In this setting, the fractal dimension of $K$ and of $\mu$ is defined by $D=\frac{\log m}{-\log a}$, and it is known that their Hausdorff dimension is also equal to $D$.

To prove Theorem 1.3, we shall relate $K$ and $\mu$ to a symbolic model, easier to work with.

\subsubsection{Symbolic Bernoulli measures}

Consider $\Sigma:=\{1,2, \ldots, m\}^{\mathbb{N}}$ endowed with the metric $\rho_{a}$ defined as follows. For each $x=\left(x_{i}\right)_{i \geq 0}$ and $y=\left(y_{i}\right)_{i \geq 0}$ in $\Sigma$, we set $i(x, y)=\min \left\{i \geq 0 \mid x_{i} \neq y_{i}\right\} \in \mathbb{N} \cup\{\infty\}$ and $\rho_{a}(x, y)=a^{i(x, y)} \in[0,1]$. The metric $\rho_{a}$ is ultrametric: $\rho_{a}(x, z) \leq \max \left\{\rho_{a}(x, y), \rho_{a}(y, z)\right\}$, and $\left(\Sigma, \rho_{a}\right)$ has Hausdorff dimension $D=\frac{\log m}{-\log a}$. 
Given a finite word $\lambda=\lambda_{0} \ldots \lambda_{j}$ with letters $\lambda_{i} \in\{1, \ldots, m\}$, we define the cylinder

$$
C_{\lambda}:=\left\{x=\left(x_{i}\right)_{i \geq 0} \mid x_{i}=\lambda_{i} \forall i \leq j\right\}
$$

Cylinders are exactly the balls of radius $a^{j}$ and they are clopen sets. The set of finite words is denoted by $\Lambda$, and $\Lambda_{j}$ is the subset of words with $j+1$ letters. When $\lambda \in \Lambda_{j}$, we say that $C_{\lambda}$ is a depth- $j$ cylinder.

We shall consider the uniform Bernoulli product measure, which is an analogue of the uniform measure on a self-similar fractal:

$$
\left(\sum_{j=1}^{m} \frac{1}{m} \delta_{j}\right)^{\otimes \mathbb{N}} \in \mathcal{P}(\Sigma) .
$$

Theorem 2.6. Let $\mu$ be the Bernoulli product measure on the symbolic space $\left(\Sigma, \rho_{a}\right)$ on $m$ letters and let $\left(X_{k}\right)_{k \geq 0}$ be i.i.d. random variables of law $\mu$, and recall $D=\frac{\log m}{-\log a}$. Then

$$
\mathbb{E}\left[\mathrm{W}_{1}\left(\hat{\mu}_{n}, \mu\right)\right] \asymp \begin{cases}\frac{1}{\sqrt{n}} & \text { if } D<2, \\ \frac{\log n}{\sqrt{n}} & \text { if } D=2, \\ \frac{1}{n^{\frac{1}{D}}} & \text { if } D>2 .\end{cases}
$$

Proof. The proof of the upper bound follows the proof of Theorem 2.1, replacing the dyadic decomposition by the partition in cylinders. Briefly, one considers a 1-Lipschitz function $f: \Sigma \rightarrow \mathbb{R}$ and approximates it by linear combinations of the $\psi_{\lambda}:=\mathbf{1}_{C_{\lambda}}$ where $\lambda$ runs through $\Lambda$. We set $f_{-1}=f(111 \ldots)$; once $f_{j-1}$ is defined for some $j \geq 0$, for each $\lambda \in \Lambda_{j-1}$ we set $\alpha(\lambda)=\left(f-f_{j-1}\right)(\lambda \cdot 11 \ldots)$ and $f_{j}=f_{j-1}+\sum_{\lambda \in \Lambda_{j-1}} \alpha(\lambda) \psi_{\lambda}$. Then we have $\alpha(\lambda) \lesssim a^{j}$ for all $\lambda \in \Lambda_{j}$ (when $j \geq 1$ ) and $\left\|f-f_{j-1}\right\|_{\infty} \lesssim a^{j}$. The same computations as in the proof of Theorem 2.1 yields for all $J \in \mathbb{N}$ :

$$
\mathbb{E}\left[\mathrm{W}_{1}\left(\hat{\mu}_{n}, \mu\right)\right] \lesssim a^{J}+\sum_{j=1}^{J} \frac{a^{j} m^{\frac{j}{2}}}{\sqrt{n}} .
$$

In the case $D=2$, we have $a^{\frac{1}{2}}=1$ and we can take $J=\log _{\frac{1}{a}} n+O(1)$ to obtain $\mathbb{E}\left[\mathrm{W}_{1}\left(\hat{\mu}_{n}, \mu\right)\right] \lesssim \log (n) / \sqrt{n}$. When $D<2$, we have $a m^{\frac{1}{2}}<1$ and it suffices to let $J \rightarrow \infty$ to obtain $\mathbb{E}\left[\mathrm{W}_{1}\left(\hat{\mu}_{n}, \mu\right)\right] \lesssim 1 / \sqrt{n}$. When $D>2$, we have $a m^{\frac{1}{2}}>1$ and we get

$$
\mathbb{E}\left[\mathrm{W}_{1}\left(\hat{\mu}_{n}, \mu\right)\right] \lesssim a^{J}+\frac{\left(a m^{\frac{1}{2}}\right)^{J}}{\sqrt{n}}
$$

we are lead to choose $J$ so that both terms are of the same order, and $J=\frac{\log n}{\log m}+O(1)$ makes them both $O\left(n^{\frac{\log n}{\log m}}\right)=O\left(n^{-\frac{1}{D}}\right)$, as expected. 
Let us now turn to the lower bound, where our main interest lies. The core of the argument is to prove that for all $n>m$,

$$
\mathbb{E}\left[\mathrm{W}_{1}\left(\hat{\mu}_{n}, \mu\right)\right] \geq \frac{C}{\sqrt{n}}+a \mathbb{E}\left[\mathrm{W}_{1}\left(\hat{\mu}_{\left\lfloor\frac{n}{m}\right\rfloor}, \mu\right)\right]
$$

First, we reduce to the case when $n$ is a multiple of $m$. Let $n^{\prime}=m\left\lfloor\frac{n}{m}\right\rfloor$; we have $n^{\prime}=n+O(1)$ and

$$
\mathrm{W}_{1}\left(\frac{1}{n} \sum_{k=1}^{n} \delta_{X_{k}}, \frac{1}{n^{\prime}} \sum_{k=1}^{n^{\prime}} \delta_{X_{k}}\right) \leq \frac{C}{n}
$$

(use the coupling that leaves a mass $1 / n$ at each $X_{k}$ for $k \leq n^{\prime}$, and spreads equally the mass of the remaining $O(1)$ points among the $n^{\prime}$ first ones.) Up to including this error $O(1 / n)=O(1 / \sqrt{n})$ in the above constant $C$, we can replace $n$ by $n^{\prime}$, i.e. assume that $n / m$ is an integer.

Now, we consider in each depth-0 cylinder $C_{\lambda}$ (where $\lambda \in \Lambda_{0}=\{1, \ldots, m\}$ ) the proportion $P_{\lambda}$ of the $\left(X_{k}\right)$ that belong to $C_{\lambda}$ (from now on, all cylinders to be considered shall be of depth 0 , and all $\lambda$ are in $\Lambda_{0}$ ). Let us set $t_{\lambda}=\left|P_{\lambda}-\frac{1}{m}\right|$ and $t=\sum_{\lambda} t_{\lambda}$; this random variable will have a central importance. The random variables $n P_{\lambda}$ each have a binomial law of parameters $(n, 1 / m)$, so that $\mathbb{E}\left[t_{\lambda}\right] \asymp 1 / \sqrt{n}$ and $\mathbb{E}[t] \asymp 1 / \sqrt{n}$. Consider the set of pairs of points differing in their first coordinates,

$$
T:=\bigcup_{\lambda \neq \lambda^{\prime} \in \Lambda_{0}} C_{\lambda} \times C_{\lambda^{\prime}}=\left\{(x, y) \in \Sigma \times \Sigma \mid x_{0} \neq y_{0}\right\}=\left\{(x, y) \in \Sigma \times \Sigma \mid \rho_{a}(x, y)=1\right\}
$$

For any transport plan $\gamma$ from $\mu$ to $\hat{\mu}_{n}$, we have $\gamma(T) \geq t$; and since cylinders have diameter $a$ but pariwise distance $1>a$, if $\gamma$ is optimal then $\gamma(T)=t$ (from now on, we fix an optimal $\gamma$ ).

Construct a new family of $n$ random points $\left(Y_{\lambda, i}\right)_{\lambda \in \Lambda_{1}, 1 \leq i \leq n / m}$ as follows. We impose $Y_{\lambda, i} \in C_{\lambda}$, so that $C_{\lambda}$ contains exactly $n / m$ points, which are drawn as follows:

- if $P_{\lambda}<1 / m$, the first $P_{\lambda}$ points $Y_{\lambda, i}$ are the $X_{k} \in C_{\lambda}$ and the remaining ones are drawn uniformly with law the restriction of $\mu_{\Sigma}$ to $C_{\lambda}$, independently of all other random variables,

- if $P_{\lambda}=1 / m$, the $Y_{\lambda, i}$ are exactly the $X_{k} \in C_{\lambda}$,

- if $P \lambda>1 / m$, the $Y_{\lambda, i}$ are $n / m$ of the $X_{k} \in C_{\lambda}$, chosen uniformly and independently of everything else.

Let $\bar{\mu}_{n}=\frac{1}{n} \sum_{\lambda, i} \delta_{Y_{\lambda, i}}$. For each $\lambda$, the $\left(Y_{\lambda, i}\right)_{0 \leq i \leq \frac{n}{m}}$ are independent and uniformly distributed on $C_{\lambda}$, which is a copy of $\Sigma$ scaled by a factor $a$. Since the diameter of each $C_{\lambda}$ is lesser than the distance between any two of them, and since both $\bar{\mu}_{n}$ and $\mu$ give the same mass $1 / m$ to all $C_{\lambda}$, an optimal transport plan $\eta$ from $\mu$ to $\bar{\mu}_{n}$ does not move mass between different cylinders, i.e. $\eta(T)=0$. As consequence, we have

$$
\mathbb{E}\left[W_{1}\left(\bar{\mu}_{n}, \mu\right)\right]=\sum_{\lambda \in \Lambda_{0}} \frac{a}{m} \mathbb{E}\left[W_{1}\left(\hat{\mu}_{\frac{n}{m}}, \mu\right)\right]=a \mathbb{E}\left[W_{1}\left(\hat{\mu}_{\frac{n}{m}}, \mu\right)\right] .
$$

We shall now construct a transport plan $\zeta$ from $\mu$ to $\bar{\mu}_{n}$ that will provide a lower bound on $\mathrm{W}_{1}\left(\bar{\mu}_{n}, \mu\right)$ involving $\mathrm{W}_{1}\left(\hat{\mu}_{n}, \mu\right)$. The idea is to start from $\gamma$ and replace all movements of mass between different cylinders by movements of mass inside the cylinders, while minimizing the total variation between $\zeta$ and $\gamma$. Now we make this idea more formal.

Decompose $\gamma$ in two parts: $\gamma_{\mid T}$ (of total mass $t$ ) and $\gamma^{\prime}=\gamma-\gamma_{\mid T}$, which is the restriction of $\gamma$ on the pair of points that are in the same cylinder, or equivalently that are at distance at most $a$. Let $\mu^{\prime}, \hat{\mu}_{n}^{\prime}$ be the marginals of $\gamma^{\prime}$ and $\mu^{\prime \prime}, \hat{\mu}_{n}^{\prime \prime}$ be the marginals of $\gamma_{\mid T}$ (one could say that $\mu^{\prime \prime}$ is the part of the mass of $\mu$ that is moved by $\gamma$ between different cylinders). By construction of the $Y_{\lambda, i}$, whenever $P_{\lambda} \leq \frac{1}{m}$ the restrictions of $\hat{\mu}_{n}, \hat{\mu}_{n}^{\prime}$ and $\bar{\mu}_{n}$ to $C_{\lambda}$ satisfy $\hat{\mu}_{n \mid C_{\lambda}}=\hat{\mu}_{n \mid C_{\lambda}}^{\prime} \leq \bar{\mu}_{n \mid C_{\lambda}}$; while when $P_{\lambda}>\frac{1}{m}$ the mass of $\hat{\mu}_{n \mid C_{\lambda}}^{\prime m}$ could be spread over all 
$X_{k} \in C_{\lambda}$, which outnumber the $\left(Y_{\lambda, i}\right)_{1 \leq i \leq \frac{n}{m}}$. In the former case, let $\zeta_{\lambda}^{\prime}=\gamma_{\mid C_{\lambda} \times C_{\lambda}}^{\prime}$; it is a transport plan between $\mu_{\mid C_{\lambda}}^{\prime} \leq \mu_{\mid C_{\lambda}}$ and $\hat{\mu}_{n \mid C_{\lambda}} \leq \bar{\mu}_{n \mid C_{\lambda}}$. We can thus extend $\zeta_{\lambda}^{\prime}$ into a transport plan $\zeta_{\lambda}$ from $\mu_{\mid C_{\lambda}}$ to $\bar{\mu}_{n \mid C_{\lambda}}$ (extending meaning that $\zeta_{\lambda}^{\prime} \leq \zeta_{\lambda}$ ). In the latter case, let $\zeta_{\lambda}$ be a transport plan between $\mu_{\mid C_{\lambda}}$ and $\bar{\mu}_{n \mid C_{\lambda}}$ (both have mass $1 / m)$ which has at much mass in common with $\gamma_{C_{\lambda} \times C \lambda}^{\prime}$, i.e. there is a positive measure $\xi$ of mass $\frac{1}{m}-t_{\lambda}$ such that $\xi \leq \zeta_{y}$ and $\xi \leq \gamma_{\mid C_{\lambda} \times C_{\lambda}}^{\prime}$.

We obtain a transport plan $\zeta=\sum_{\lambda} \zeta_{\lambda}$ which, compared to $\gamma$, gains a cost of $t$ by not moving this amount of mass between different cylinders, and loses a cost of at by moving the same amount of mass within cylinders to adjust the difference between $\hat{\mu}_{n}$ and $\bar{\mu}_{n}$. In consequence, we have

$$
\mathrm{W}_{1}\left(\bar{\mu}_{n}, \mu\right) \leq \mathrm{W}_{1}\left(\hat{\mu}_{n}, \mu\right)-t+a t .
$$

Taking an expectation and using (2.9), $a<1$ and $\mathbb{E}[t] \asymp 1 / \sqrt{n}$, we obtain (2.8).

Now, let us deduce the desired lower bounds from (2.8). Let $\left(n_{j}\right)_{0 \leq j \leq J}$ be the sequence defined by $n_{0}=n$ and $n_{j+1}=\left\lfloor\frac{n_{j}}{m}\right\rfloor$, up to the first index $J$ such that $n_{J} \leq m$. Then $J=\log _{m} n+O(1)$ and $\mathrm{W}_{1}\left(\hat{\mu}_{n_{J}}, \mu\right) \asymp 1$, implying

$$
\begin{aligned}
\mathrm{W}_{1}\left(\hat{\mu}_{n}, \mu\right) \geq & \frac{C}{\sqrt{n}}+\frac{a C}{\sqrt{n / m}}+\cdots+\frac{a^{J-1} C}{\sqrt{n / m^{J-1}}}+\mathrm{W}_{1}\left(\hat{\mu}_{n_{J}}, \mu\right) \\
& \gtrsim \begin{cases}\frac{1}{\sqrt{n}} & \text { if } a \sqrt{m}<1, \\
\frac{J}{\sqrt{n}} & \text { if } a \sqrt{m}=1, \\
\frac{(a \sqrt{m})^{J}}{\sqrt{n}} & \text { if } a \sqrt{m}>1 .\end{cases}
\end{aligned}
$$

Recalling $D=\log m /(-\log a)$, these are the desired bounds.

Proof of Theorem 1.3. Here $\mu$ is the uniform measure on the self-similar fractal $K$ defined by the IFS $\Phi$. Let denote by $\mu_{\Sigma}$ the uniform Bernoulli product measure on $\Sigma$.

Given $x \in \Sigma$, the sequence of compact sets $\phi_{x_{0}} \phi_{x_{1}} \ldots \phi_{x_{i}}(K)$ is decreasing with diameter converging to 0 as $i \rightarrow \infty$; its intersection thus consists in a single element denoted by $\pi(x)$. It is well-known and easy to check directly that the map $\pi: \Sigma \rightarrow \mathbb{R}^{d}$ defined in this way induces a bi-Lipschitz homeomorphism from $\Sigma$ onto $K$ (both assumptions, that maps are similarities of equal ratios and the strong separation condition, are crucial here). Moreover, $\mu$ is the push-forward by $\pi$ of $\mu_{\Sigma}$. The random variables $Z_{k}=\pi\left(X_{k}\right)$ are thus i.i.d. with law $\mu_{\Sigma}$, and Theorem 1.3 reduces to Theorem 2.6; to take into account the parameter $q$, simply observe that $\rho_{a}^{q}=\rho_{a^{q}}$.

\section{WAVElet DECOMPOSITION AND CONVERGENCE AGAinst REGUlaR TEST FUNCTIONS}

\subsection{Wavelet decomposition}

Let us give a short account of the results about wavelets we will use (see e.g. Meyer's book [21] for proofs and references).

It will be convenient to use wavelets of compact support with arbitrary regularity $\mathcal{C}^{\mathrm{r}}$, whose construction is due to Daubechies [6]. The construction yields compactly supported functions $\phi, \psi^{\epsilon}: \mathbb{R}^{d} \rightarrow \mathbb{R}$ where $\epsilon$ takes any of $2^{d}-1$ values $\left(\epsilon \in E:=\{0,1\}^{d} \backslash\{(0,0, \ldots, 0)\}\right)$, with particular properties of which only those we will use will be described. 
One defines from these "father and mother" wavelets a larger family of wavelets by

$$
\begin{aligned}
& \phi_{\tau}(x)=\phi(x-\tau), \quad\left(\tau \in \mathbb{Z}^{d}\right) \\
& \psi_{\lambda}(x)=2^{\frac{d j}{2}} \psi^{\epsilon}\left(2^{j} x-\tau\right), \quad\left(\lambda=(j, \tau, \epsilon) \in \Lambda=\mathbb{Z} \times \mathbb{Z}^{d} \times E\right) ;
\end{aligned}
$$

one important property of the construction is that the union of $\left(\phi_{\tau}\right)_{\tau \in \mathbb{Z}^{d}}$ and $\left(\psi_{\lambda}\right)_{\lambda \in \Lambda}$ form an orthonormal basis of $L^{2}\left(\mathbb{R}^{d}\right)$. For $f \in L^{2}\left(\mathbb{R}^{d}\right)$ we can thus write

$$
f=\sum_{\tau \in \mathbb{Z}^{d}}\left\langle f, \phi_{\tau}\right\rangle \phi_{\tau}+\sum_{j=0}^{\infty} \sum_{\lambda \in \Lambda_{j}}\left\langle f, \psi_{\lambda}\right\rangle \psi_{\lambda}
$$

where $\Lambda_{j}=\{j\} \times \mathbb{Z}^{d} \times E$ and $\langle\cdot, \cdot\rangle$ denotes the $L^{2}$ scalar product (with respect to Lebesgue measure).

One stunning property is that many functional spaces can be characterized in term of the wavelet coefficients $\alpha(\lambda)=\left\langle f, \psi_{\lambda}\right\rangle$ and $\beta(\tau)=\left\langle f, \phi_{\tau}\right\rangle$. We shall only use upper bounds on the $\alpha(\lambda)$ and $\beta(\tau)$ in a specific case.

The Hölder space $\mathcal{C}^{\mathrm{s}}$ is defined as the space of $k$ times continuously differentiable with $\gamma$-Hölder partial derivatives of order $k$, with $k$ a non-negative integer, $\gamma \in(0,1]$ and $k+\gamma=s\left(e . g . \mathcal{C}^{1}\right.$ is the space of Lipschitz functions, $\mathcal{C}^{3 / 2}$ the space of once continuously differentiable functions with $1 / 2$-Hölder first-order partial derivatives, $\mathcal{C}^{5}$ is the space of four-times continuously differentiable functions with Lipschitz fourth-order partial derivatives, etc.). Note that "1-Hölder", meaning "Lipschitz", could be slightly enlarged to "Zygmund" (and should, if one is interested in two-sided bounds), but we need not enter this subtlety here.

The space $\mathcal{C}^{\text {s }}$ is endowed with the norm

$$
\|f\|_{\mathcal{C}^{s}}=\max _{j \in\{0, \ldots, k\}} \max _{\omega \in\{1, \ldots, d\}^{j}}\left\|\frac{\partial^{j} f}{\partial x_{\omega_{1}} \cdots \partial x_{\omega_{j}}}\right\|_{\star}
$$

where the decomposition $s=k+\gamma$ is defined as above and $\|\cdot\|_{\star}$ is the uniform norm if $j<k$ and the maximum of the uniform norm and the $\gamma$-Hölder constant if $j=k$. We denote by $\mathcal{C}_{1}^{\mathrm{s}}$ the set of functions with $\mathcal{C}^{\mathrm{s}}$ norm at most 1.

If the regularity of the wavelets is larger than the regularity of the considered Hölder space $(r>s)$ then

$$
\begin{array}{ll}
|\beta(\tau)| \leq C_{d, s}\|f\|_{\infty} & \forall \tau \in \mathbb{Z}^{d} \\
|\alpha(\lambda)| \leq C_{d, s}\|f\|_{\mathcal{C}^{s}} 2^{-\frac{d j}{2}} 2^{-j s} & \forall \lambda \in \Lambda_{j},
\end{array}
$$

where the constant $C_{d, s}$ depends implicitely on the choice of father and mother wavelets $\phi$ and $\psi^{\epsilon}$; but we can fix for each $s$ such a choice with suitable regularity, e.g. $r=s+1$ and the constants then truly depends only on $d$ and $s$. The $\mathcal{C}^{\mathrm{s}}$ norm in the $\alpha(\lambda)$ coefficient could be relaxed to the "regularity part" of the norm but we do not use this.

Note that the explicit computation of these constants would in particular need a very fine analysis of the chosen wavelet construction, and I do not know whether such a task has been conducted.

\subsection{Decomposition of regular functions}

Let us now use wavelet decomposition to prove good convergence properties for the empirical measure against smooth enough test functions; the strategy is similar to the one used in Section 2. We assume here that $\left(X_{k}\right)_{k} \geq 0$ is a sequence of i.i.d. random variables whose law $\mu$ is supported on a bounded set $\Omega \subset \mathbb{R}^{d}\left(e . g . \Omega=[0,1]^{\bar{d}}\right)$; note that $\mathcal{C}_{1}^{\mathrm{s}}=\mathcal{C}_{1}^{\mathrm{s}}\left(\mathbb{R}^{d}\right)$ makes no reference to $\Omega$. We consider a fixed family of wavelet of regularity $r>s$ as in Section 3.1; all constants $C$ below implicitly depend on $d, s$ and $\Omega$ (only through its diameter). 
Since the wavelets have compact support, there exist some constant $C$ such that for each $j$ :

- for each point $x \in \Omega$, there are at most $C$ different $\lambda$ corresponding to a $\psi_{\lambda}$ that does not vanish at $x$; the set of those $\lambda$ is denoted by $\Lambda_{j}(x) \subset \Lambda_{j}$,

- the union $\Lambda_{j}(\Omega):=\bigcup_{x \in \Omega} \lambda_{j}(x)$ has at most $C 2^{d j}$ elements.

We denote by $Z$ the set of parameters $\tau \in \mathbb{Z}^{d}$ corresponding to a $\phi_{\tau}$ whose support intersects $\Omega$ (observe that $Z$ is finite).

We fix a function $f \in \mathcal{C}_{1}^{\mathrm{s}}$ and decompose it in our wavelet basis:

$$
f=\sum_{\tau \in \mathbb{Z}^{d}} \beta(\tau) \phi_{\tau}+\sum_{j=0}^{\infty} \sum_{\lambda \in \Lambda_{j}} \alpha(\lambda) \psi_{\lambda}
$$

with

$$
\begin{array}{ll}
|\beta(\tau)| \lesssim 1 & \forall \tau \in \mathbb{Z}^{d} \\
|\alpha(\lambda)| \lesssim 2^{-\frac{d j}{2}} 2^{-j s} & \forall \lambda \in \Lambda_{j} .
\end{array}
$$

Cutting the second term of the decomposition to some depth $J$ we get:

$$
f=\sum_{\tau \in Z} \beta(\tau) \phi_{\tau}+\sum_{j=0}^{J} \sum_{\lambda \in \Lambda_{j}} \alpha(\lambda) \psi_{\lambda}+g
$$

where

$$
g=\sum_{\tau \notin Z} \beta(\tau) \phi_{\tau}+\sum_{j>J} \sum_{\lambda \in \Lambda_{j}} \alpha(\lambda) \psi_{\lambda}
$$

Using the bound on the $\alpha$ coefficients and the formula (3.1) for $\psi_{\lambda}$, we get:

$$
\left\|g \mathbf{1}_{\Omega}\right\|_{\infty} \lesssim 2^{-s J}
$$

and it follows:

$$
\left|\hat{\mu}_{n}(f)-\mu(f)\right| \lesssim 2^{-J s}+\sum_{\tau \in Z}\left|\hat{\mu}_{n}\left(\phi_{\tau}\right)-\mu\left(\phi_{\tau}\right)\right|+\sum_{j=0}^{J} \sum_{\lambda \in \Lambda_{j}(\Omega)} 2^{-\left(\frac{d}{2}+s\right) j}\left|\hat{\mu}_{n}\left(\psi_{\lambda}\right)-\mu\left(\psi_{\lambda}\right)\right|
$$

where the right-hand side does not depend on $f$. Taking a supremum and an expectation, it then comes:

$$
\mathbb{E}\left[\left\|\hat{\mu}_{n}-\mu\right\|_{\mathcal{C}_{1}^{\mathrm{s}}}\right] \lesssim 2^{-s J}+\sum_{\tau \in Z} \mathbb{E}\left[\left|\hat{\mu}_{n}\left(\phi_{\tau}\right)-\mu\left(\phi_{\tau}\right)\right|\right]+\sum_{j=0}^{J} \sum_{\lambda \in \Lambda_{j}(\Omega)} 2^{-\left(\frac{d}{2}+s\right) j} \mathbb{E}\left[\left|\hat{\mu}_{n}\left(\psi_{\lambda}\right)-\mu\left(\psi_{\lambda}\right)\right|\right]
$$

and to conclude, we simply need to estimate the last two terms above.

\subsection{Convergence for basis elements}

Lemma 3.1. We have

$$
\sum_{\tau \in Z} \mathbb{E}\left[\left|\hat{\mu}_{n}\left(\phi_{\tau}\right)-\mu\left(\phi_{\tau}\right)\right|\right] \lesssim \frac{1}{\sqrt{n}}
$$




$$
\text { and } \quad \sum_{\lambda \in \Lambda_{j}(\Omega)} \mathbb{E}\left[\left|\hat{\mu}_{n}\left(\psi_{\lambda}\right)-\mu\left(\psi_{\lambda}\right)\right|\right] \lesssim \frac{2^{d j}}{\sqrt{n}} .
$$

Proof. For each $\tau \in Z$, the random variable $\hat{\mu}_{n}\left(\phi_{\tau}\right)$ is the average of $n$ independent identically distributed, bounded random variables of expectation $\mu\left(\phi_{\tau}\right)$, so that $\mathbb{E}\left[\left|\hat{\mu}_{n}\left(\phi_{\tau}\right)-\mu\left(\phi_{\tau}\right)\right|\right] \leq C / \sqrt{n}$. Since $Z$ is finite, the first claim is proved.

To prove the second claim, we cannot argue in the exact same way because $\psi_{\lambda}$ depends on $j$. To ease notation we introduce $\bar{\psi}_{\lambda}:=2^{-\frac{d j}{2}} \psi_{\lambda}$ and $Y_{\lambda}:=\hat{\mu}_{n}\left(\bar{\psi}_{\lambda}\right)-\mu\left(\bar{\psi}_{\lambda}\right)$, and recall that $\bar{\psi}_{\lambda}$ is bounded independently of $j$. Observe that $Y_{\lambda}$ is the average of the $n$ centered, i.i.d. random variables $\bar{\psi}_{\lambda}\left(X_{k}\right)-\mu\left(\bar{\psi}_{\lambda}\right)$ for $k$ in $\{1, \ldots, n\}$ and that

$$
\operatorname{Var}\left(\bar{\psi}_{\lambda}\left(X_{k}\right)-\mu\left(\bar{\psi}_{\lambda}\right)\right)=\mathbb{E}\left[\bar{\psi}_{\lambda}\left(X_{k}\right)^{2}\right]-\mu\left(\bar{\psi}_{\lambda}\right)^{2} \leq \mathbb{P}\left[X_{k} \in \operatorname{supp} \psi_{\lambda}\right]\left\|\bar{\psi}_{\lambda}\right\|_{\infty}^{2} \leq C p_{\lambda}
$$

where $p_{\lambda}$ is the mass given by $\mu$ to the support of $\psi_{\lambda}$. We thus have $\operatorname{Var}\left(Y_{\lambda}\right) \leq C p_{\lambda} / n$. Also, a bounded number of different $\bar{\psi}_{\lambda}\left(\lambda \in \Lambda_{j}\right)$ are non-zero at any point $x \in \Omega$, so that $\sum_{\lambda \in \Lambda_{j}(\Omega)} p_{\lambda} \lesssim 1$. It follows

$$
\sum_{\lambda \in \Lambda_{j}(\Omega)} \operatorname{Var}\left(Y_{k}\right) \lesssim \frac{1}{n}
$$

Now it comes

$$
\begin{aligned}
\sum_{\lambda \in \Lambda_{j}(\Omega)} \mathbb{E}\left[\left|\hat{\mu}_{n}\left(\psi_{\lambda}\right)-\mu\left(\psi_{\lambda}\right)\right|\right] & =2^{\frac{d j}{2}} \sum_{\lambda \in \Lambda_{j}(\Omega)} \mathbb{E}\left[\left|Y_{\lambda}\right|\right] \\
& \leq 2^{\frac{d j}{2}} \sum_{\lambda \in \Lambda_{j}(\Omega)} \sqrt{\mathbb{E}\left[Y_{\lambda}^{2}\right]} \\
& \leq 2^{\frac{d j}{2}} \sqrt{\left|\Lambda_{j}(\Omega)\right|} \sqrt{\sum_{\lambda \in \Lambda_{j}(\Omega)} \operatorname{Var}\left(Y_{\lambda}\right)} \\
& \lesssim \frac{2^{d j}}{\sqrt{n}}
\end{aligned}
$$

Remark 3.2. Lemma 3.1 is the only place where we use that the $\left(X_{k}\right)_{k \in \mathbb{N}}$ are i.i.d. The method can therefore be applied to any stochastic process satisfying the conclusion of Lemma 3.1.

\subsection{Conclusion of the proof}

Plugging Lemma 3.1 into (3.2) yields

$$
\mathbb{E}\left[\left\|\hat{\mu}_{n}-\mu\right\|_{\mathcal{C}_{1}^{\mathrm{s}}}\right] \lesssim 2^{-J s}+\frac{1}{\sqrt{n}} \sum_{j=0}^{J}\left(2^{\frac{d}{2}-s}\right)^{j}
$$

and we get the same trichotomy as before. If $s>d / 2$, then we can let $J \rightarrow \infty$ to obtain

$$
\mathbb{E}\left[\left\|\hat{\mu}_{n}-\mu\right\|_{\mathcal{C}_{1}^{\mathrm{s}}}\right] \leq \frac{C}{\sqrt{n}}
$$


If $s=d / 2$ we can take $J$ such that $2^{-J s} \simeq 1 / \sqrt{n}$ and get

$$
\mathbb{E}\left[\left\|\hat{\mu}_{n}-\mu\right\|_{\mathcal{C}_{1}^{\mathrm{s}}}\right] \leq C \frac{\log n}{\sqrt{n}} .
$$

If $s<d / 2$ we can choose $J$ such that $2^{J} \simeq n^{\frac{1}{d}}$ to get

$$
\mathbb{E}\left[\left\|\hat{\mu}_{n}-\mu\right\|_{\mathcal{C}_{1}^{s}}\right] \leq \frac{C}{n^{s / d}}
$$

ending the proof of Theorem 1.4.

\section{MARKOV CHAINS}

In this section we assume $\left(X_{k}\right)_{k \geq 0}$ is a Markov chain on a bounded domain; since we will use Fourier series, it will make things simpler to embed this domain into a torus, so we assume $\Omega \subset \mathbb{T}^{d}=\mathbb{R}^{d} / \mathbb{Z}^{d}$ (we do not lose generality in doing so, as scaling down $\Omega$ makes it possible to make the embedding isometric). We still denote by $\|x-y\|$ the distance between two points induced by the Euclidean norm.

Our main assumption is that the iterated transition kernel of $\left(X_{k}\right)_{k \geq 0}$, defined by

$$
m_{x}(A)=\mathbb{P}\left(X_{k+1} \in A \mid X_{k}=x\right) \quad m_{x}^{t}(A)=\mathbb{P}\left(X_{k+t} \in A \mid X_{k}=x\right)
$$

is exponentially contracting in $\mathrm{W}_{1}$, i.e. there are constants $D \geq 1$ and $\theta \in(0,1)$ such that

$$
\mathrm{W}_{1}\left(m_{x}^{t}, m_{y}^{t}\right) \leq D \theta^{t}\|x-y\| .
$$

Let us denote by $\mathrm{L}$ the Markov operator and $\mathrm{L}^{*}$ be its dual acting on probability measures, i.e.

$$
\mathrm{L} f(x)=\int f(y) \mathrm{d} m_{x}(y) \quad \mathrm{L}^{*} \nu(f)=\int \mathrm{L} f \mathrm{~d} \nu=\int m_{x}(f) \mathrm{d} \mu(x)
$$

(in other words, whenever $X_{k}$ has law $\nu, X_{k+1}$ has law $\mathrm{L}^{*} \nu$ ). The linearity of the Kantorovich transport problem (1.1) from which $\mathrm{W}_{1}$ is defined enables one to rewrite (4.1) as

$$
\mathrm{W}_{1}\left(\mathrm{~L}^{* t} \nu_{0}, \mathrm{~L}^{* t} \nu_{1}\right) \leq D \theta^{t} \mathrm{~W}_{1}\left(\nu_{0}, \nu_{1}\right)
$$

so that there is a unique stationary measure $\mu$, and the law of $X_{k}$ converges exponentially fast (in $\mathrm{W}_{1}$ ) to $\mu$, whatever the law of $X_{0}$ is.

We shall prove Theorem 1.1, which we restate for convenience; recall that $s$ is positive integer.

Theorem 4.1. There exists a constant $C=C(\Omega, d, D, s)>0$ such that for all $n \geq N$, letting $\bar{n}=(1-\theta) n$, we have

$$
\mathbb{E}\left[\left\|\hat{\mu}_{n}-\mu\right\|_{\mathcal{C}_{1}^{\mathrm{s}}}\right] \leq C \begin{cases}\frac{(\log \bar{n})^{\frac{d}{2 s+1}}}{\sqrt{\bar{n}}} & \text { when } s>d / 2, \\ \frac{\log \bar{n}}{\sqrt{\bar{n}}} & \text { when } s=d / 2, \\ \frac{(\log \bar{n})^{d-2 s+\frac{s}{d}}}{\bar{n}^{\frac{s}{d}}} & \text { when } s<d / 2 .\end{cases}
$$


Following the decomposition method, we shall find a suitable decomposition basis for any $f \in \mathcal{C}_{1}^{\mathrm{s}}$, seeking for a compromise between precision of a truncated decomposition and number of basis elements. Here using wavelets seems inefficient, as we do not have a precise enough analogue of Lemma 3.1, which uses independence to take advantage of the localization property of wavelets; without this, the number and size of the $\psi_{\lambda}$ are overwhelming. We shall use Fourier series instead, as they will be more easily controlled under our assumptions. For simplicity we consider complex-valued functions here, and denote the Fourier basis by $e_{k}(x):=e^{2 i \pi k \cdot x}$ where $k \in \mathbb{Z}^{d}$ and the dot $\cdot$ denotes the canonical inner product.

The key is thus to control $\left|\hat{\mu}_{n}\left(e_{k}\right)-\mu\left(e_{k}\right)\right|$; our hypothesis may seem perfectly suited to this since $e_{k}$ is Lipschitz, but its Lipschitz constant grows too rapidly with $k$ for a direct approach to be efficient. We shall combine the following two observations (the first of which is pretty trivial, the second of which is folklore).

Lemma 4.2. For all $\alpha \in(0,1)$, we have the following control of $e_{k}$ 's $\alpha$-Hölder constant:

$$
\operatorname{Hol}_{\alpha}\left(e_{k}\right) \lesssim|k|_{\infty}^{\alpha}
$$

where $|k|_{\infty}=\max \left\{k_{i}: i \in\{1, \ldots, d\}\right\}$.

Proof. We have $\operatorname{Lip}\left(e_{k}\right) \leq 2 \pi \sqrt{d}|k|_{\infty}$ and $\left\|e_{k}\right\|_{\infty} \leq 1$ so that for all $x \neq y \in \mathbb{T}^{d}$ :

$$
\frac{\left|e_{k}(x)-e_{k}(y)\right|}{\|x-y\|^{\alpha}} \leq \min \left(\frac{2}{\|x-y\|^{\alpha}}, 2 \pi \sqrt{d}|k|_{\infty}\|x-y\|^{1-\alpha}\right) \leq 2 \pi^{\alpha} d^{\frac{\alpha}{2}}|k|_{\infty}^{\alpha} .
$$

Lemma 4.3. For all $\alpha \in(0,1]$, denoting by $\mathrm{W}_{\alpha}$ the $\alpha$-Wasserstein metric (i.e. either the 1 -Wasserstein metric associated with the modified distance $\|\cdot\|^{\alpha}$ or equivalently the metric obtained by duality with functions of $\alpha$ Hölder constant 1), we have

$$
\mathrm{W}_{\alpha}\left(\mathrm{L}_{0}^{* t} \nu_{0}, \mathrm{~L}_{0}^{* t} \nu_{1}\right) \leq D^{\alpha} \theta^{\alpha t} \mathrm{~W}_{\alpha}\left(\nu_{0}, \nu_{1}\right) .
$$

As a consequence, for all $\alpha$-Hölder functions $f: \Omega \rightarrow \mathbb{C}$ and all $\ell, m \in \mathbb{N}$ it holds

$$
\begin{aligned}
\left|\mathbb{E}\left[f\left(X_{\ell}\right)\right]-\mu(f)\right| & \lesssim \operatorname{Hol}_{\alpha}(f) \theta^{\alpha \ell} \\
\left|\mathbb{E}\left[f\left(X_{m}\right) f\left(X_{\ell}\right)\right]-\mathbb{E}\left[f\left(X_{m}\right)\right] \mathbb{E}\left[f\left(X_{\ell}\right)\right]\right| & \lesssim \operatorname{Hol}_{\alpha}(f)^{2} \theta^{\alpha|m-\ell|}
\end{aligned}
$$

where the implied constants depends only on $\Omega$ and the constant $D$ in (4.1).

Proof. By linearity we only have to check (4.4) when $\nu_{0}=\delta_{x}$ and $\nu_{1}=\delta_{y}$ for some $x, y \in \Omega$, and by concavity

$$
\mathrm{W}_{\alpha}\left(\mathrm{L}^{* t} \delta_{x}, \mathrm{~L}^{* t} \delta_{y}\right) \leq\left(\mathrm{W}_{1}\left(\mathrm{~L}^{* t} \delta_{x}, \mathrm{~L}^{* t} \delta_{y}\right)\right)^{\alpha} \leq D^{\alpha} \theta^{\alpha t}\|x-y\|^{\alpha}=D^{\alpha} \theta^{\alpha t} \mathrm{~W}_{\alpha}\left(\delta_{x}, \delta_{y}\right) .
$$

To prove convergence toward the average and decay of correlation, we first use the contraction and that $\mu$ is the stationary measure to get

$$
\begin{aligned}
\left|\mathrm{L}^{t} f(x)-\mu(f)\right| & =\left|\int \mathrm{L}^{t} f \mathrm{~d} \delta_{x}-\int f \mathrm{~d} \mu\right| \\
& =\left|\int f \mathrm{~d}\left(\mathrm{~L}^{* t} \delta_{x}\right)-\int f \mathrm{~d}\left(\mathrm{~L}^{* t} \mu\right)\right| \\
& \leq \operatorname{Hol}_{\alpha}(f) \mathrm{W}_{\alpha}\left(\mathrm{L}^{* t} \delta_{x}, \mathrm{~L}^{* t} \mu\right) \\
& \leq \operatorname{Hol}_{\alpha}(f) D^{\alpha} \theta^{\alpha t} \mathrm{~W}_{\alpha}\left(\delta_{x}, \mu\right) \\
\left|\mathrm{L}^{t} f(x)-\mu(f)\right| & \lesssim \operatorname{Hol}_{\alpha}(f) \theta^{\alpha t} .
\end{aligned}
$$


Assuming without lost of generality $\mu(f)=0$ we have $\|f\|_{\infty} \lesssim \operatorname{Hol}_{\alpha}(f)(\mu(f)=0$ implies that $f$ takes both non-positive and non-negative values, and $\Omega$ is bounded). Assume further $m \geq \ell$ and write $m=\ell+t$. Combining all previous observations we get:

$$
\begin{aligned}
\left\|\mathrm{L}^{t} f\right\|_{\infty} & \lesssim \operatorname{Hol}_{\alpha}(f) \theta^{\alpha t} \\
\left|\mathbb{E}\left[f\left(X_{m}\right)\right]\right| & =\left|\mathbb{E}\left[\mathrm{L}^{m} f\left(X_{0}\right)\right]\right| \\
& \lesssim \operatorname{Hol}_{\alpha}(f) \theta^{\alpha m} \\
\left|\mathbb{E}\left[f\left(X_{\ell}\right)\right]\right| & \lesssim \operatorname{Hol}_{\alpha}(f) \theta^{\alpha \ell} \\
\left|\mathbb{E}\left[f\left(X_{m}\right) f\left(X_{\ell}\right)\right]\right| & =\left|\mathbb{E}\left[\mathrm{L}^{t} f\left(X_{\ell}\right) f\left(X_{\ell}\right)\right]\right| \\
& \lesssim\left\|\mathrm{L}^{t} f\right\|_{\infty} \mathbb{E}\left[\left|f\left(X_{\ell}\right)\right|\right] \\
& \lesssim \operatorname{Hol}_{\alpha}(f)^{2} \theta^{\alpha t}
\end{aligned}
$$

and the conclusion follows.

We deduce the following from these two Lemmas.

Corollary 4.4. For all $k, \alpha, n$ it holds

$$
\mathbb{E}\left[\left|\hat{\mu}_{n}\left(e_{k}\right)-\mu\left(e_{k}\right)\right|^{2}\right] \lesssim \frac{|k|_{\infty}^{2 \alpha}}{\left(1-\theta^{\alpha}\right) n}
$$

Proof. We set $\bar{e}_{k}:=e_{k}-\mu\left(e_{k}\right)$, which has the same Hölder constant than $e_{k}$ and vanishing average. We have:

$$
\begin{aligned}
\mathbb{E}\left[\left|\hat{\mu}_{n}\left(e_{k}\right)-\mu\left(e_{k}\right)\right|^{2}\right] & =\frac{1}{n^{2}} \sum_{1 \leq \ell, m \leq n} \mathbb{E}\left[\bar{e}_{k}\left(X_{\ell}\right) \bar{e}_{k}\left(X_{m}\right)\right] \\
& \lesssim \frac{1}{n^{2}} \sum_{\ell=1}^{n} \sum_{m \geq \ell} \mathbb{E}\left[\bar{e}_{k}\left(X_{\ell}\right) \bar{e}_{k}\left(X_{m}\right)\right] \\
& \lesssim \frac{1}{n^{2}} \sum_{\ell=1}^{n} \sum_{m \geq \ell} \operatorname{Hol}_{\alpha}\left(\bar{e}_{k}\right)^{2} \theta^{\alpha(m-\ell)} \\
& \lesssim \frac{1}{n^{2}} \sum_{\ell=1}^{n} \frac{|k|_{\infty}^{2}}{1-\theta^{\alpha}}=\frac{|k|_{\infty}^{2 \alpha}}{\left(1-\theta^{\alpha}\right) n} .
\end{aligned}
$$

Fix some threshold $J \geq 3$ and some exponent $\alpha \in(0,1]$, to be determined explicitly later on.

Let $f: \mathbb{T}^{d} \rightarrow \mathbb{R}$ be in $\mathcal{C}_{1}^{\mathrm{s}}$. From the multidimensional version of Jackson's theorem [25], we know that there is a trigonometric polynomial $T_{J}(f)$ which is a linear combination of the $e_{k}$ for $|k|_{\infty} \leq J$, such that

$$
\left\|f-T_{J}(f)\right\|_{\infty} \lesssim \frac{1}{J^{s}}
$$

We have no clear control on the coefficient of this optimal trigonometric polynomial, which need not be the Fourier coefficients of $f$. But it is also known that the Fourier series of $f$ is within a factor $\simeq\|f\|_{\infty}(\log J)^{d}$ of the best approximation (see [20] for an optimal constant), so that denoting by $F_{J}(f):=\sum_{|k|_{\infty} \leq J} \hat{f}_{k} e_{k}$ the 
$J$-truncation of the Fourier series of $f$, we get

$$
\left\|f-F_{J}(f)\right\|_{\infty} \lesssim \frac{(\log J)^{d}}{J^{s}}
$$

We can assume $\hat{f}_{0}=0$ by translating $f$, and what precedes yields:

$$
\begin{aligned}
&\left|\hat{\mu}_{n}(f)-\mu(f)\right| \leq\left|\hat{\mu}_{n}(f)-\hat{\mu}_{n}\left(F_{J}(f)\right)\right|+\left|\hat{\mu}_{n}\left(F_{J}(f)\right)-\mu\left(F_{J}(f)\right)\right|+\left|\mu\left(F_{J}(f)\right)-\mu(f)\right| \\
& \leq 2\left\|f-F_{J}(f)\right\|_{\infty}+\sum_{0<|k|_{\infty} \leq J}\left|\hat{f}_{k}\right|\left|\hat{\mu}_{n}\left(e_{k}\right)-\mu\left(e_{k}\right)\right| \\
& \lesssim \frac{(\log J)^{d}}{J^{s}}+\left(\sum_{0<|k|_{\infty} \leq J}\left|\hat{f}_{k}\right|^{2}|k|_{\infty}^{2 s}\right)^{\frac{1}{2}}\left(\sum_{0<|k|_{\infty} \leq J} \frac{\left|\hat{\mu}_{n}\left(e_{k}\right)-\mu\left(e_{k}\right)\right|^{2}}{|k|_{\infty}^{2 s}}\right)^{\frac{1}{2}} \\
& \lesssim \frac{(\log J)^{d}}{J^{s}}+\|f\|_{H^{s}}\left(\sum_{0<|k|_{\infty} \leq J} \frac{\left|\hat{\mu}_{n}\left(e_{k}\right)-\mu\left(e_{k}\right)\right|^{2}}{|k|_{\infty}^{2 s}}\right)^{\frac{1}{2}} \\
&\left|\hat{\mu}_{n}(f)-\mu(f)\right| \lesssim \frac{(\log J)^{d}}{J^{s}}+\left(\sum_{0<|k|_{\infty} \leq J} \frac{\left|\hat{\mu}_{n}\left(e_{k}\right)-\mu\left(e_{k}\right)\right|^{2}}{|k|_{\infty}^{2 s}}\right)^{\frac{1}{2}}
\end{aligned}
$$

where the right-hand side does not depend on $f$ in any way; above

$$
\|f\|_{H^{s}}:=\left(\sum_{k \in \mathbb{Z}^{d}}\left|\hat{f}_{k}\right|^{2}\left(1+|k|_{\infty}^{2}\right)^{s}\right)^{\frac{1}{2}}
$$

is the Sobolev norm, controlled by the $\mathcal{C}^{\text {s }}$ norm when $s \in \mathbb{N}$ (Sobolev functions of order $s$ are also those who have weak derivatives in $L^{2}$ up to order $s$, and by the Rademacher theorem Lipschitz functions have weak derivatives in $\left.L^{\infty}\right)$.

Remark 4.5. At line (4.5), one could be tempted to use the bound $\left|\hat{f}_{k}\right| \lesssim|k|_{\infty}^{-s}$ instead of using the CauchySchwarz inequality; this ultimately leads to a weaker bound.

Taking a supremum and an expectation in (4.6) and using concavity, it comes:

$$
\begin{aligned}
\mathbb{E}\left[\left\|\hat{\mu}_{n}-\mu\right\|_{\mathcal{C}_{1}^{s}}\right] & \lesssim \frac{(\log J)^{d}}{J^{s}}+\left(\sum_{0<|k|_{\infty} \leq J} \frac{\mathbb{E}\left[\left|\hat{\mu}_{n}\left(e_{k}\right)-\mu\left(e_{k}\right)\right|^{2}\right]}{|k|_{\infty}^{2 s}}\right)^{\frac{1}{2}} \\
& \lesssim \frac{(\log J)^{d}}{J^{s}}+\left(\sum_{0<|k|_{\infty} \leq J} \frac{|k|^{2 \alpha}}{\left(1-\theta^{\alpha}\right) n|k|_{\infty}^{2 s}}\right)^{\frac{1}{2}} \\
& \lesssim \frac{(\log J)^{d}}{J^{s}}+\left(\sum_{\ell=1}^{J} \frac{\ell^{d-1+2 \alpha-2 s}}{\left(1-\theta^{\alpha}\right) n}\right)^{\frac{1}{2}}
\end{aligned}
$$


Choose now $\alpha=1 / \log J$ so that $\ell^{2 \alpha} \lesssim 1$ for all $\ell \in\{1, \ldots, J\}$, use $1-\theta^{\alpha} \geq \alpha(1-\theta)$ and set $\bar{n}:=(1-\theta) n$ to obtain

$$
\mathbb{E}\left[\left\|\hat{\mu}_{n}-\mu\right\|_{\mathcal{C}_{1}^{\mathrm{s}}}\right] \lesssim \frac{(\log J)^{d}}{J^{s}}+\sqrt{\frac{\log J}{\bar{n}}}\left(\sum_{\ell=1}^{J} \ell^{d-1-2 s}\right)^{\frac{1}{2}}
$$

For $s<d / 2$, we get:

$$
\mathbb{E}\left[\left\|\hat{\mu}_{n}-\mu\right\|_{\mathcal{C}_{1}^{\mathrm{s}}}\right] \lesssim \frac{(\log J)^{d}}{J^{s}}+\frac{(\log J)^{\frac{1}{2}} J^{\frac{d}{2}-s}}{\sqrt{\bar{n}}}
$$

Trying to balance the contribution of the two terms, we first see that taking $J \simeq \bar{n}^{\frac{1}{d}}$ would optimize the power of $\bar{n}$ in the final expression; refining to $J=(\log \bar{n})^{\beta} \bar{n}^{\frac{1}{d}}$, developing and ignoring lower order terms shows that the choice $\beta=2-\frac{1}{d}$ optimizes the final power of $\log \bar{n}$, and we thus set $J=\left\lfloor(\log \bar{n})^{2-\frac{1}{d}} \bar{n}^{\frac{1}{d}}\right\rfloor$. It then comes:

$$
\mathbb{E}\left[\left\|\hat{\mu}_{n}-\mu\right\|_{\mathcal{C}_{1}^{\S}}\right] \lesssim \frac{(\log \bar{n})^{d-2 s+\frac{s}{d}}}{\bar{n}^{\frac{s}{d}}}
$$

For $2 s=d$ we get

$$
\mathbb{E}\left[\left\|\hat{\mu}_{n}-\mu\right\|_{\mathcal{C}_{1}^{s}}\right] \lesssim \frac{(\log J)^{d}}{J^{s}}+\frac{\log J}{\sqrt{\bar{n}}}
$$

and taking $J=\left\lfloor\bar{n} \frac{1}{2 s}(\log \bar{n})^{(d-1) / s}\right\rfloor$ yields

$$
\mathbb{E}\left[\mathrm{W}_{1}\left(\hat{\mu}_{n}, \mu\right)\right] \lesssim \frac{\log \bar{n}}{\sqrt{\bar{n}}}
$$

Finally, for $s>d / 2$ we get

$$
\mathbb{E}\left[\left\|\hat{\mu}_{n}-\mu\right\|_{\mathcal{C}_{1}^{\mathrm{s}}}\right] \lesssim \frac{(\log J)^{d}}{J^{s}}+\frac{(\log J)^{\frac{1}{2}}}{\sqrt{\bar{n}}}
$$

and taking $J=\left\lfloor\bar{n}^{\frac{1}{2 s}}(\log \bar{n})^{\frac{d}{s+1 / 2}}\right\rfloor$ yields

$$
\mathbb{E}\left[\left\|\hat{\mu}_{n}-\mu\right\|_{\mathcal{C}_{1}^{\mathbb{s}}}\right] \lesssim \frac{(\log \bar{n})^{\frac{d}{2 s+1}}}{\sqrt{\bar{n}}}
$$

ending the proof of Theorem 1.1.

\section{Concentration near the expectation}

Let us detail how classical bounded martingale difference methods can be used to prove that the empirical measure concentrates very strongly around its expectation. When $\left(X_{k}\right)_{k \geq 0}$ are independent identically distributed, this is long-known (see [26], and also [30] for more general Wasserstein metrics $\mathrm{W}_{p}, p \geq 1$ ). In the case of Markov chains, such arguments have been developed notably in [4], [7] and, in a dynamical context, [5]. Our approach is very similar and thus cannot pretend to much novelty, but we write it down both to show how to handle functional spaces more general than just Lipschitz and Hölder, and because it seems not easy to find it in this form in the literature. 
Note that other previous results may pop into mind, but are not actually applicable here. A first line of works consider strong mixing assumptions, where the law of $X_{k}$ converges toward $\mu$ in total variation (see e.g. [19] and references therein). Such methods do not apply here since the law of $X_{k}$ itself (and not only $\hat{\mu}_{n}$ ) can be purely atomic for all $k$ even when $\mu$ is absolutely continuous. Another line of work is concerned with concentration bounds under the same kind of "geometric ergodicity" / "curvature bounds" hypotheses we make here [14], [15], [24] - however these works are concerned with the concentration of empirical averages around the expectation, not with the deviation of the empirical measure from the stationary measure (see the discussion below equation $(1.4)$ ).

The fundamental result to be used is the Azuma-Hoeffding inequality, which we recall.

Theorem 5.1 (Azuma-Hoeffding inequality). Let $Y$ be a random variable, let

$$
\{\varnothing, \Omega\}=\mathscr{B}_{0} \subset \mathscr{B}_{1} \subset \cdots \subset \mathscr{B}_{n}=\mathscr{B}(\Omega)
$$

be a filtration and for each $k \in \llbracket 1, n \rrbracket$ set $\Delta_{k}=\mathbb{E}\left[Y \mid \mathscr{B}_{k}\right]-\mathbb{E}\left[Y \mid \mathscr{B}_{k-1}\right]$. Assume that for all $k$ and some numbers $a_{k} \in \mathbb{R}, c_{k}>0$ we have $\Delta_{k} \in\left[a_{k}, a_{k}+c_{k}\right]$ almost surely. Then for all $t>0$,

$$
\mathbb{P}[Y \geq \mathbb{E}[Y]+t] \leq \exp \left(-\frac{2 t^{2}}{\sum_{k} c_{k}^{2}}\right)
$$

\subsection{The independent case}

In the case of i.i.d. random variables, the Azuma-Hoeffding inequality famously yields the following concentration inequality.

Theorem 5.2 (McDiarmid's inequality). Let $F: \Omega^{n} \rightarrow \mathbb{R}$ be a function such that for some $c_{1}, \ldots, c_{n}$ and all $k \in \llbracket 1, n \rrbracket$ and all $\left(x_{1}, \ldots, x_{n}, x_{k}^{\prime}\right) \in \Omega^{n+1}$ it holds

$$
\left|F\left(x_{1}, \ldots, x_{k}, \ldots, x_{n}\right)-F\left(x_{1}, \ldots, x_{k}^{\prime}, \ldots, x_{n}\right)\right| \leq c_{k} .
$$

Let $\left(X_{k}\right)_{1 \leq k \leq n}$ be a sequence of independent random variables. Then for all $t>0$ it holds

$$
\mathbb{P}\left[F\left(X_{1}, \ldots, X_{n}\right) \geq \mathbb{E}\left[F\left(X_{1}, \ldots, X_{n}\right)\right]+t\right] \leq \exp \left(-\frac{2 t^{2}}{\sum_{k} c_{k}^{2}}\right)
$$

Applying this to

$$
F\left(X_{1}, \ldots, X_{n}\right)=\left\|\hat{\mu}_{n}-\mu\right\|_{\mathscr{F}}=\sup _{f \in \mathscr{F}}\left|\frac{1}{n} \sum_{k=1}^{n} f\left(X_{k}\right)-\mu(f)\right|
$$

we can take

$$
c_{k}=\frac{1}{n} \sup _{f \in \mathscr{F}, x, x^{\prime} \in \Omega}\left|f(x)-f\left(x^{\prime}\right)\right|=: \frac{1}{n} \operatorname{osc}(\mathscr{F})
$$

and it comes

$$
\mathbb{P}\left[F\left(X_{1}, \ldots, X_{n}\right) \geq \mathbb{E}\left[F\left(X_{1}, \ldots, X_{n}\right)\right]+t\right] \leq \exp \left(-\frac{2 n t^{2}}{\operatorname{osc}(\mathscr{F})^{2}}\right)
$$


For example if $\mathscr{F} \subset \operatorname{Lip}_{1}(\Omega)\left(\right.$ e.g. $\left.\mathscr{F}=\mathcal{C}_{1}^{\text {s }}\right)$ we have $\operatorname{osc}(\mathscr{F}) \leq \operatorname{diam} \Omega$; if moreover $\Omega=[0,1]^{d}$ it thus comes

$$
\mathbb{P}\left[\left\|\hat{\mu}_{n}-\mu\right\|_{\mathscr{F}} \geq \mathbb{E}\left[\left\|\hat{\mu}_{n}-\mu\right\|_{\mathscr{F}}\right]+t\right] \leq \exp \left(-\frac{2}{d} \cdot n t^{2}\right)
$$

This, combined with Theorem 1.4, yields good concentration estimates.

Corollary 5.3. If $\left(X_{k}\right)_{k \geq 0}$ are i.i.d.random variables with law $\mu$, then for all $s \geq 1$, for some constant $C=$ $C(d, s)>0$ (not depending upon $\mu$ ), all integer $n \geq 2$ and all $M \geq C$ we have:

- if $s>d / 2$

$$
\mathbb{P}\left[\left\|\hat{\mu}_{n}-\mu\right\|_{\mathcal{C}_{1}^{\mathrm{s}}} \geq \frac{M}{\sqrt{n}}\right] \leq e^{-\frac{2}{d}(M-C)^{2}},
$$

- if $s=d / 2$

$$
\mathbb{P}\left[\left\|\hat{\mu}_{n}-\mu\right\|_{\mathcal{C}_{1}^{\mathrm{s}}} \geq \frac{M \log n}{\sqrt{n}}\right] \leq e^{-\frac{2}{d}(M-C)^{2}(\log n)^{2}},
$$

- if $s<d / 2$

$$
\mathbb{P}\left[\left\|\hat{\mu}_{n}-\mu\right\|_{\mathcal{C}_{1}^{\mathrm{s}}} \geq \frac{M}{n^{\frac{s}{d}}}\right] \leq e^{-\frac{2}{d}(M-C)^{2} n^{1-2 s / d}}
$$

Similarly, with Theorem 1.2 we can obtain entirely explicit, non-asymptotic concentration bounds.

\subsection{Markov chains}

To tackle Markov chains we will need some hypothesis to replace independence; we choose a framework that covers the case of $\mathrm{W}_{1}$, but also more general dual metrics $\|\cdot\|_{\mathscr{F}}$.

Assume that $\Omega$ is endowed with a metric $d$ with finite diameter ( $d$ is assumed to be lower-semi-continuous, but not necessarily to induce the given topology on $\Omega$ ). We still denote by $\operatorname{Lip}_{1}(\Omega)$ be the space of functions $\Omega \rightarrow \mathbb{R}$ which are 1-Lipschitz with respect to $d$.

Let $\left(X_{k}\right)_{>0}$ be a Markov chain on $\Omega$ which is exponentially contracting (see the beginning of Sect. 4) with constant $D$ and rate $\theta$, in the metric $d$ instead of the euclidean norm; this can be rewritten in a coupling formulation as follows: for all $x, x^{\prime} \in \Omega$, all $i, t \in \mathbb{N}$ there are random variables $\left(X_{k}^{\prime}\right)_{k \geq i}$ with the same law as $\left(X_{k}\right)_{k \geq i}$ and such that for all $t$ :

$$
\mathbb{E}\left[d\left(X_{i+t}, X_{i+t}^{\prime}\right) \mid X_{i}=x, X_{i}^{\prime}=x^{\prime}\right] \leq D \theta^{t} d\left(x, x^{\prime}\right) .
$$

Note that the flexibility in the choice of $d$ enables to include uniformly ergodic Markov chains in this framework, simply by taking $d=\mathbf{1}_{\neq}$, i.e. $d(x, y)=0$ if $x=y$ and $d(x, y)=1$ otherwise. by

Given a multivariate function $\Phi: \Omega^{n} \rightarrow \mathbb{R}^{n}$, we define as usual the coordinate-wise Lipschitz constants of $\Phi$

$$
\Lambda_{i}(\Phi)=\sup _{x_{1}, \ldots, x_{n} \in \Omega, x_{i}^{\prime} \neq x_{i}} \frac{\left|\Phi\left(x_{1}, \ldots, x_{i}, \ldots, x_{n}\right)-\Phi\left(x_{1}, \ldots, x_{i}^{\prime}, \ldots, x_{n}\right)\right|}{d\left(x_{i}, x_{i}^{\prime}\right)}
$$

and we say that $\Phi$ is separately Lipschitz if $\Lambda_{i}(\Phi)<\infty$ for all $i$ (when $d=\mathbf{1}_{\neq}$, the coordinate-wise Lipschitz constant become the coordinate-wise oscillations). 
Theorem 5.4. Let $\left(X_{k}\right)_{k \geq 1}$ be a Markov chain whose kernel is exponentially contracting with constant $D \geq 1$ and rate $\theta \in(0,1)$, with respect to a lower-semi-continuous distance $d$ on $\Omega$ giving it finite diameter $\operatorname{diam}(\Omega)$.

Let $n \in \mathbb{N}$ and $\Phi: \Omega^{n} \rightarrow \mathbb{R}$ be separately Lipschitz with constants $\Lambda_{i}(\Phi) \leq \Lambda$. Then

$$
\mathbb{P}\left[\Phi\left(X_{1}, \ldots, X_{n}\right) \geq \mathbb{E}\left[\Phi\left(X_{1}, \ldots, X_{n}\right)\right]+t\right] \leq \exp \left(-\frac{(1-\theta)^{2} t^{2}}{2 n D^{2} \operatorname{diam}(\Omega)^{2} \Lambda^{2}}\right)
$$

Proof. We set $X=\left(X_{1}, \ldots, X_{n}\right)$ and $X_{i: j}=\left(X_{i}, \ldots, X_{j}\right)$ (meaning the empty family whenever $j<i$ ).

We shall apply the Azuma-Hoeffding inequality with the filtration $\mathscr{B}_{k}=\sigma\left(X_{1}^{k}\right)$, leaving us with the task of bounding the oscillations $c_{k}$ of the random variable

$$
\Delta_{k}=\mathbb{E}\left[\Phi(X) \mid X_{1: k}\right]-\mathbb{E}\left[\Phi(X) \mid X_{1: k-1}\right]
$$

Given an arbitrary $x_{1: k}=\left(x_{1}, \ldots, x_{k}\right) \in \Omega^{k}$ and $x_{k}^{\prime} \in \Omega$ we set

$$
V_{k}\left(x_{1: k}, x_{k}^{\prime}\right)=\mathbb{E}\left[\Phi(X) \mid X_{1: k}=x_{1: k}\right]-\mathbb{E}\left[\Phi(X) \mid X_{1: k-1}=x_{1: k-1}, X_{k}=x_{k}^{\prime}\right]
$$

so that $c_{k}=\sup V_{k}-\inf V_{k} \leq 2\left\|V_{k}\right\|_{\infty}$. Let $\left(X_{i}^{\prime}\right)_{i \geq k}$ be a copy of $\left(X_{i}\right)_{i \geq k}$ as in the definition of exponential contraction; then

$$
\begin{aligned}
V_{k}\left(x_{1: k}, x_{k}^{\prime}\right) & =\mathbb{E}\left[\Phi\left(x_{1: k-1}, X_{k: n}\right) \mid X_{k}=x_{k}\right]-\mathbb{E}\left[\Phi\left(x_{1: k-1}, X_{k: n}^{\prime}\right) \mid X_{k}^{\prime}=x_{k}^{\prime}\right] \\
& =\sum_{i=k}^{n} \mathbb{E}\left[\Phi\left(x_{1: k-1}, X_{k: i}, X_{i+1: n}^{\prime}\right)-\Phi\left(x_{1: k-1}, X_{k: i-1}, X_{i: n}^{\prime}\right) \mid X_{k}=x_{k}, X_{k}^{\prime}=x_{k}^{\prime}\right] \\
\left|V_{k}\left(x_{1}^{k}, x_{k}^{\prime}\right)\right| & \leq \sum_{i=k}^{n} \mathbb{E}\left[\Lambda d\left(X_{i}, X_{i}^{\prime}\right) \mid X_{k}=x_{k}, X_{k}^{\prime}=x_{k}^{\prime}\right] \\
& \leq D \Lambda d\left(x_{k}, x_{k}^{\prime}\right) \sum_{i=k}^{\infty} \theta^{i-k} \\
c_{k} & \leq 2 C \Lambda \operatorname{diam}(\Omega) /(1-\theta) .
\end{aligned}
$$

Applying the Azuma-Hoeffding inequality finishes the proof.

Remark 5.5. The above inequality is probably not optimal; one can expect to improve the rate, either by moving the constant 2 from the denominator to the numerator, or by replacing $(1-\theta)^{2}$ by $(1-\theta)$ (probably with another constant).

As soon as $\mathscr{F} \subset \operatorname{Lip}_{1}(\Omega)\left(\right.$ e.g. $\left.\mathscr{F}=\mathcal{C}_{1}^{\mathrm{s}}\right)$, Theorem 5.4 applies to

$$
\Phi(X)=\left\|\hat{\mu}_{n}-\mu\right\|_{\mathscr{F}}=\sup _{f \in \mathscr{F}} \frac{1}{n} \sum_{k=1}^{n} f\left(X_{k}\right)-\mu(f)
$$

with $\Lambda=\frac{1}{n}$, yielding

$$
\mathbb{P}\left[\left\|\hat{\mu}_{n}-\mu\right\|_{\mathscr{F}} \geq \mathbb{E}\left[\left\|\hat{\mu}_{n}-\mu\right\|_{\mathscr{F}}\right]+t\right] \leq \exp \left(-\frac{(1-\theta)^{2}}{2 D^{2} \operatorname{diam}(\Omega)^{2}} \cdot n t^{2}\right)
$$

i.e., as in the independent case, subgaussian concentration. Corollary 1.5 follows. 


\section{REFERENCES}

[1] M. Ajtai, J. Komlós and G. Tusnády, On optimal matchings. Combinatorica 4 (1984) 259-264.

[2] L. Ambrosio, F. Stra and D. Trevisan, A PDE approach to a 2-dimensional matching problem. Probab. Theory Related Fields 173 (2019) 433-477.

[3] E. Boissard and T. Le Gouic, On the mean speed of convergence of empirical and occupation measures in Wasserstein distance. Ann. Inst. Henri Poincaré Probab. Stat. 50 (2014) 539-563.

[4] J.-R. Chazottes and F. Redig, Concentration inequalities for Markov processes via coupling. Electron. J. Probab. 14 (2009) $1162-1180$.

[5] J.-R. Chazottes and S. Gouëzel, Optimal concentration inequalities for dynamical systems. Commun. Math. Phys. 316 (2012) 843-889.

[6] I. Daubechies, Orthonormal bases of compactly supported wavelets. Commun. Pure Appl. Math. 41 (1988) 909-996.

[7] J. Dedecker and X. Fan, Deviation inequalities for separately Lipschitz functionals of iterated random functions. Stochastic Process. Appl. 125 (2015) 60-90.

[8] J. Dedecker and F. Merlevède, Behavior of the empirical Wasserstein distance in $\mathrm{R}^{d}$ under moment conditions. Electron. J. Probab. 24 (2019).

[9] S. Dereich, M. Scheutzow and R. Schottstedt, Constructive quantization: approximation by empirical measures. Ann. Inst. Henri Poincaré Probab. Stat. 49 (2013) 1183-1203.

[10] R.M. Dudley, Real analysis and probability. Vol. 74 of Cambridge Studies in Advanced Mathematics. Revised reprint of the 1989 original. MR 1932358. Cambridge University Press, Cambridge (2002).

[11] A. Eberle, Reflection couplings and contraction rates for diffusions. Probab. Theory Related Fields 166 (2016) 851-886.

[12] N. Fournier and A. Guillin, On the rate of convergence in Wasserstein distance of the empirical measure. Probab. Theory Related Fields 162 (2015) 707-738.

[13] J.E. Hutchinson, Fractals and self-similarity. Indiana Univ. Math. J. 30 (1981) 713-747.

[14] A. Joulin, A new Poisson-type deviation inequality for Markov jump processes with positive Wasserstein curvature. Bernoulli 15 (2009) 532-549.

[15] A. Joulin and Y. Ollivier, Curvature, concentration and error estimates for Markov chain Monte Carlo. Ann. Probab. 38 (2010) 2418-2442.

[16] B.R. Kloeckner, An optimal transportation approach to the decay of correlations for non-uniformly expanding maps. Ergodic Theory Dyn. Syst. 40 (2020) 714-750.

[17] B. Kloeckner, Effective Berry-Esseen and concentration bounds for Markov chains with a spectral gap. Ann. Appl. Probab. 29 (2019) 1778-1807.

[18] B.R. Kloeckner, A.O. Lopes and M. Stadlbauer, Contraction in the Wasserstein metric for some Markov chains, and applications to the dynamics of expanding maps. Nonlinearity 28 (2015) 4117-4137.

[19] A. Kontorovich, Obtaining measure concentration from Markov contraction. Markov Process. Related Fields 18 (2012) 613-638.

[20] J.C. Mason, Near-best multivariate approximation by Fourier series, Chebyshev series and Chebyshev interpolation. J. Approx. Theory 28 (1980) 349-358.

[21] Y. Meyer, Wavelets and operators. Vol. 1. Cambridge University Press (1992).

[22] P. Monmarché, On $H^{1}$ and entropic convergence for contractive PDMP. Electron. J. Probab. 20 (2015).

[23] Y. Ollivier, Ricci curvature of Markov chains on metric spaces. J. Funct. Anal. 256 (2009) 810-864.

[24] D. Paulin, Mixing and concentration by Ricci curvature. J. Funct. Anal. 270 (2016) 1623-1662.

[25] M.H. Schultz, $L^{\text {inf }}$-multivariate approximation theory. SIAM J. Numer. Anal. 6 (1969) 161-183.

[26] M. Talagrand, Matching random samples in many dimensions. Ann. Appl. Probab. 2 (1992) 846-856.

[27] M. Talagrand, Sharper bounds for Gaussian and empirical processes. Ann. Probab. 22 (1994) $28-76$.

[28] A.W. van der Vaart and J.A. Wellner, Weak convergence and empirical processes. With applications to statistics. MR 1385671. Springer Series in Statistics. Springer-Verlag, New York (1996).

[29] R. van Handel, Probability in high dimension, APC 550 Lecture Notes. Princeton University (1996). Available from: http: //www.princeton.edu/ rvan/APC550.pdf.

[30] J. Weed and F. Bach, Sharp asymptotic and finite-sample rates of convergence of empirical measures in Wasserstein distance. Bernoulli 25 (2019) 2620-2648.

[31] J. Weed and Q. Berthet, Estimation of smooth densities in wasserstein distance. Preprint arXiv:1902.01778 (2019). 